\title{
The Role of the Ocean in the Global Atmospheric Sulfur Cycle
}

\author{
B. C. Nguyen, B. Bonsang, and A. Gaudry
}

Centre des Faibles Radioactivités, Laboratoire Mixte CNRS-CEA

\begin{abstract}
The background atmospheric $\mathrm{SO}_{2}$ concentration above the sea surface has been established at $0.1 \times 10^{-6} \mathrm{~g} / \mathrm{m}^{3}$ (35 pptv). Several hypotheses to explain the origin of $\mathrm{SO}_{2}$ above the ocean, far from continental sources, have been discussed. We have shown that one of the mechanisms of $\mathrm{SO}_{2}$ formation is the biogenic production of reduced organic sulfur compounds in the ocean. Dimethyl sulfide (DMS) has been observed in oceanic areas, with concentrations ranging from 11 to $1150 \times 10^{-9} \mathrm{~g} \mathrm{l}^{-1}$ in surface waters and from 0.2 to $400 \times 10^{-9} \mathrm{~g} \mathrm{~m}^{-3}$ (0.07 to $\left.144 \mathrm{pptv}\right)$ in marine atmosphere. This compound is emitted into the atmosphere and oxidized there by photochemically induced processes. That leads partly to $\mathrm{SO}_{2}$ formation, and subsequently, to sulfate. This hypothesis is corroborated by a correlation between atmospheric $\mathrm{SO}_{2}$ concentrations and the relation between atmospheric $\mathrm{SO}_{2}$ concentrations and biological activity. The flux of sulfur by this process (biological activity $\rightarrow$ organic sulfides $\rightarrow \mathrm{SO}_{2} \rightarrow \mathrm{SO}_{4}{ }^{\prime}$ ) is estimated to range between 27 and $72 \times 10^{6}$ tons of sulfur per year. This biogenic production is comparable to atmospheric sulfur production by sea spray, which is about $44 \times 10^{6} \mathrm{~T} \mathrm{~S} / \mathrm{yr}$. Therefore, the oceanic production by these two processes could be estimated as being from 71 to $116 \times 10^{6} \mathrm{~T} \mathrm{~S} / \mathrm{yr}$.
\end{abstract}

\section{INTRODUCTION}

The global sulfur budget, particularly its atmospheric component, has been discussed by numerous authors: Eriksson [1963], Robinson and Robbins [1970], Kellogg et al. [1972], Friend [1973], Granat et al. [1976]. In all of the budgets mentioned above, known sulfur sources can account for only about one half of the recognized sinks. According to these authors, the difference, which is required to balance the budget, varies between 30 and $170 \times 10^{6} \mathrm{~T} \mathrm{~S} / \mathrm{yr}$. This discrepancy indicates that natural sulfur sources are not yet well known, in particular the volcanic and oceanic sources. Several origins for this last natural source have been suggested, but data is scarce. Kellogg et al. proposed $\mathrm{H}_{2} \mathrm{~S}$ production above continents and coastal areas is the missing source, while Lovelock et al. [1972] believed organic sulfide emission from the sea surface is important. Logan et al. [1979], Torres et al. [1980], and Sze and Ko [1980] suggested that atmospheric $\mathrm{SO}_{2}$ is produced by the photooxidation of $\mathrm{COS}$ and $\mathrm{CS}_{2}$, two compounds whose sources are not yet well identified. Our goal in this paper is to present data that help to elucidate the role of the ocean in the atmospheric sulfur cycle.

\section{EXISTENCE OF SUlfur Dioxide OVER THE OCEAN}

\section{Methodology}

By using an automatic "cut-out" device controlled by a weather vane system, air samples free of contamination from the ship exhaust were collected during 24 hours and analyzed for $\mathrm{SO}_{2}$ by an adaptation of the method of West and Gaeke [1956].

The lowest detectable amount of $\mathrm{SO}_{2}$ is $0.1 \mu \mathrm{g}$, determined with sodium metabisulfite standard solutions. In order to lessen our detection limit we have used the preconcentrating device previously described [Nguyen et al., 1974a; Bonsang and Nguyen, 1978], mainly composed of an impinger and an electromagnetic device that automatically controls the solution level.

Hence the volume of air analyzed for each sample ranges

Copyright 1983 by the American Geophysical Union.

Paper number $3 \mathrm{C} 1233$.

$0148-0227 / 83 / 003 \mathrm{C}-1233 \$ 05.00$ between 4 and $5 \mathrm{~m}^{3}$, and the detection limit is about $0.02 \mu \mathrm{g}$ $\mathrm{SO}_{2} / \mathrm{m}^{3}$.

\section{Sulfur Dioxide Over the Open Ocean}

To complete our previous $\mathrm{SO}_{2}$ measurements in marine environments [Nguyen et al., 1974a], three oceanographic expeditions, described in Figure $1 a$, were undertaken in subantarctic regions and in the Indian Ocean from $5^{\circ} \mathrm{S}$ to $30^{\circ} \mathrm{S}$. In this last area the circulation of air masses is essentially zonal, from east to west; $\mathrm{SO}_{2}$ concentrations were only measured in air masses that had probably last passed over Australia several thousand kilometers away.

Figures $1 b$ and $1 c$ show a geographical distribution of 160 values of $\mathrm{SO}_{2}$ above the Indian Ocean sub-Antarctic region and the Atlantic Ocean (Table 1). The average concentration of 121 values of atmospheric $\mathrm{SO}_{2}, 0.1 \mu \mathrm{g} / \mathrm{m}^{3}$ in the Indian Ocean and the sub-Antarctic area (Figure 1c), is the same as the previous background already found above this region [Nguyen et al., 1974a].

As the basic $p \mathrm{H}$ of seawater $(\simeq 8.2)$ would prevent the direct release of dissolved $\mathrm{SO}_{2}$ to the marine atmosphere, other sources must be determined to explain the observed background concentration of $0.1 \mu \mathrm{g} / \mathrm{m}^{3}$. It is therefore relevant to discuss the various hypotheses for the occurrence of $\mathrm{SO}_{2}$ in the open ocean.

Could the $\mathrm{SO}_{2}$ be carried along great distances from remote continental sources? $\mathrm{SO}_{2}$ can be carried into the marine atmosphere either through the lower troposphere or through the upper troposphere.

Nguyen et al. [1975, 1978b] have determined a 17-hour residence time (12-hour half-life) for $\mathrm{SO}_{2}$ in the marine atmosphere of the boundary layer over the Mediterranean. A similar value was found by Prahm et al. [1976] above the North Atlantic. Furthermore, a strong decrease of $\mathrm{SO}_{2}$ concentrations has been observed over the Mediterranean. Such a decrease is due to the oxidation and dilution effects and the absorption by seawater, which represents as much as $9 \%$ to $34 \%$ of the scavenging process [Bonsang, 1980]. In fact, at 100 $\mathrm{km}$ from the coast this concentration is only one tenth of its mean coastal value $\left(5 \mu \mathrm{g} / \mathrm{m}^{3}\right)$. Even when near a polluted area such as Fos-sur-Mer, the $\mathrm{SO}_{2}$ concentrations observed 200 $\mathrm{km}$ out to sea were only $0.25 \mu \mathrm{g} / \mathrm{m}^{3}$ with winds coming from 


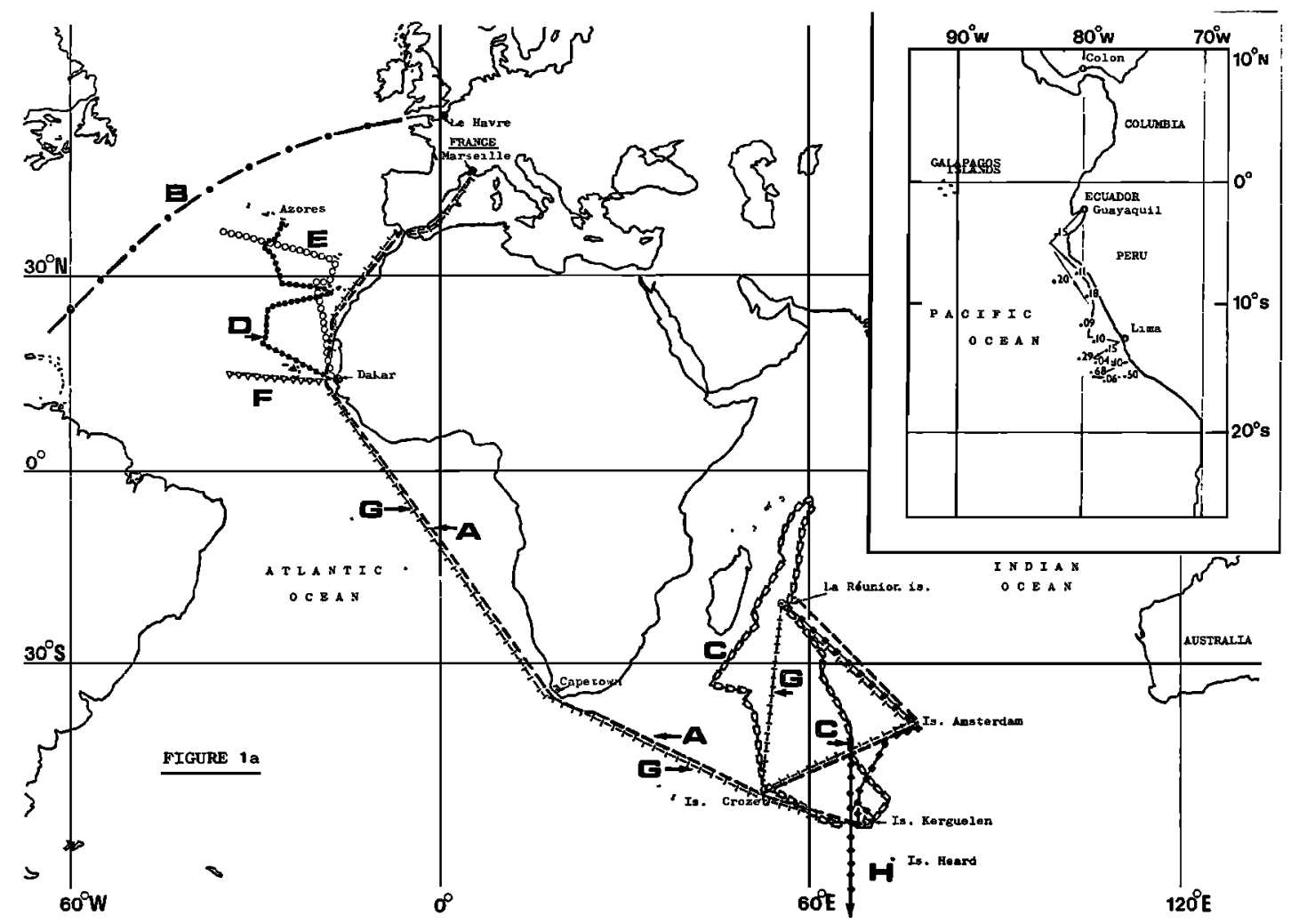

Fig. $1 a$.

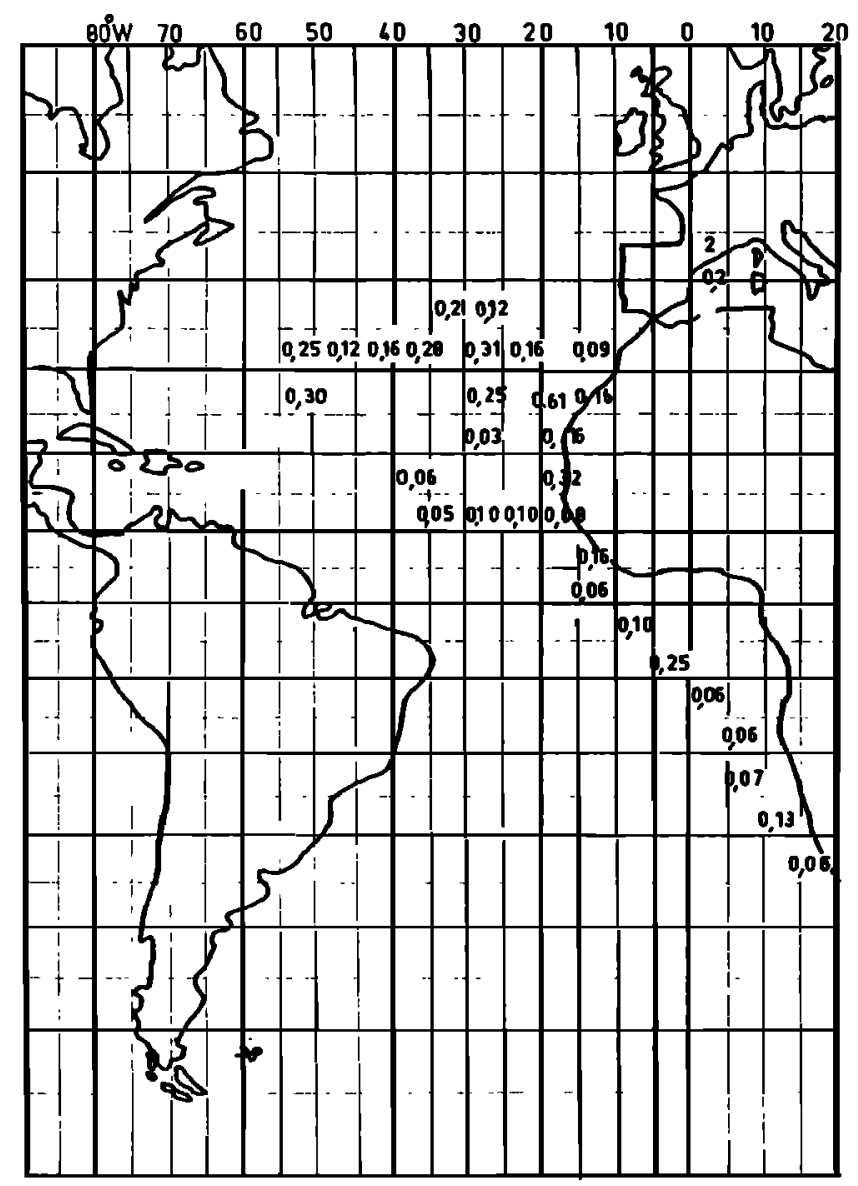

Fig. $1 b$.

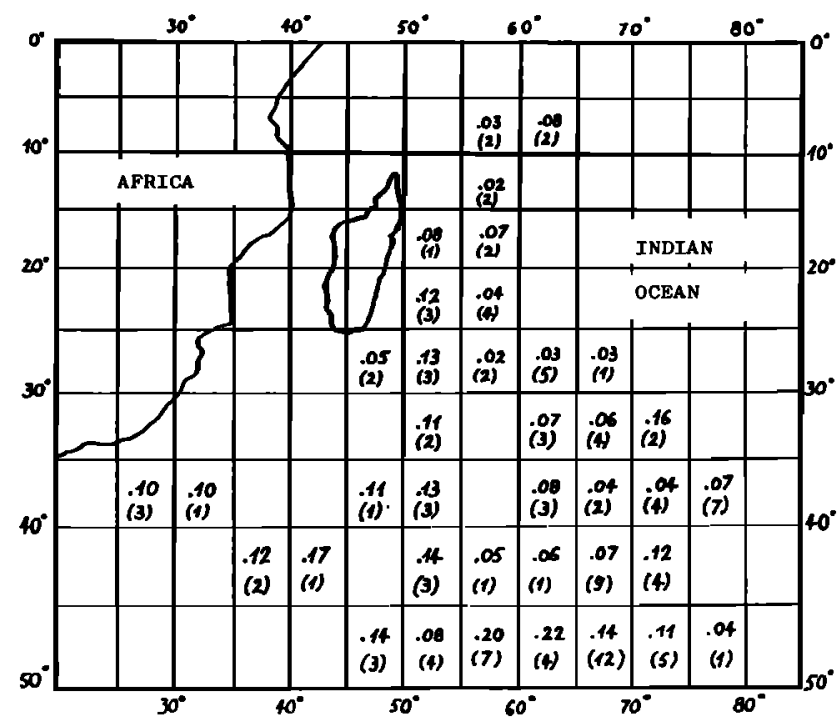

Fig. Ic.

Fig. 1. (a) Tracks for oceanographic cruises $\mathrm{A}$ to $\mathrm{H}$ in the Atlantic and Indian Ocean. Right corner: $\mathrm{SO}_{2}$ concentrations during a cruise in the Peruvian Upwelling in April 1975. (b) Mean atmospheric $\mathrm{SO}_{2}$ concentrations ( $\mu \mathrm{g} /$ $\mathrm{m}^{3}$ ) above the Atlantic Ocean and (c) above the Indian Ocean and subantarctic areas. Values in parenthesis represent the number of measurements. 
TABLE 1a. Atmospheric $\mathrm{SO}_{2}$ Concentrations: Cruise $\mathrm{A}$

Latitude

\begin{tabular}{|c|c|c|c|}
\hline Date & Start & End & $\mathrm{SO}_{2} \mu \mathrm{g} / \mathrm{m}^{3}$ \\
\hline March 11, 1973 & $42.9^{\circ} \mathrm{N}$ & $41.6^{\circ} \mathrm{N}$ & $0.096 \pm 0.008$ \\
\hline March 12,1973 & $40.5^{\circ} \mathrm{N}$ & $36.7^{\circ} \mathbf{N}$ & $0.088 \pm 0.007$ \\
\hline March 13, 1973 & $36.7^{\circ} \mathbf{N}$ & $33.2^{\circ} \mathrm{N}$ & $0.055 \pm 0.006$ \\
\hline March 14, 1973 & $33.2^{\circ} \mathrm{N}$ & $28.0^{\circ} \mathrm{N}$ & $0.094 \pm 0.007$ \\
\hline March 16,1973 & $22.6^{\circ} \mathrm{N}$ & $15.8^{\circ} \mathrm{N}$ & $0.089 \pm 0.008$ \\
\hline March 17, 1973 & $15.6^{\circ} \mathrm{N}$ & $9.7^{\circ} \mathrm{N}$ & $0.077 \pm 0.007$ \\
\hline March 18,1973 & $9.6^{\circ} \mathrm{N}$ & $4.6^{\circ} \mathrm{N}$ & $0.220 \pm 0.012$ \\
\hline March 19, 1972 & $4.5^{\circ} \mathrm{N}$ & $0.3^{\circ} \mathrm{S}$ & $0.045 \pm 0.006$ \\
\hline March 20, 1973 & $0.4^{\circ} \mathrm{S}$ & $5.4^{\circ} \mathrm{S}$ & $0.163 \pm 0.010$ \\
\hline March 21, 1973 & $5.5^{\circ} \mathrm{S}$ & $10.0^{\circ} \mathrm{S}$ & $0.440 \pm 0.020$ \\
\hline Match 22,1973 & $10.2^{\circ} \mathrm{S}$ & $15.1^{\circ} \mathrm{S}$ & $0.082 \pm 0.009$ \\
\hline March 23, 1973 & $15.1^{\circ} \mathrm{S}$ & $20.0^{\circ} \mathrm{S}$ & $0.082 \pm 0.009$ \\
\hline March 24, 1973 & $20.1^{\circ} \mathrm{S}$ & $24.7^{\circ} \mathrm{S}$ & $0.094 \pm 0.008$ \\
\hline March 25, 1973 & $24.7^{\circ} \mathrm{S}$ & $29.5^{\circ} \mathrm{S}$ & $0.106 \pm 0.010$ \\
\hline March 26, 1973 & $29.6^{\circ} \mathrm{S}$ & $34.0^{\circ} \mathrm{S}$ & $0.077 \pm 0.009$ \\
\hline March 29,1973 & $36^{\circ} 1^{\prime} \mathrm{S}$ & $38.3^{\circ} \mathrm{S}$ & $0.133 \pm 0.011$ \\
\hline March 30, 1973 & $38^{\circ} 35^{\prime} \mathrm{S}$ & $39^{\circ} 7^{\prime} \mathrm{S}$ & $0.103 \pm 0.010$ \\
\hline March 31, 1973 & $39^{\circ} 8^{\prime} \mathrm{S}$ & $42^{\circ} 9^{\prime} \mathrm{S}$ & $0.126 \pm 0.010$ \\
\hline April 1, 1973 & $43^{\circ} 0^{\prime} S$ & $46^{\circ} 1^{\prime} S$ & $0,066 \pm 0.006$ \\
\hline April 2,1973 & $46^{\circ} 15^{\prime} \mathrm{S}$ & $47^{\circ} 7^{\prime} \mathrm{S}$ & $0.094 \pm 0.010$ \\
\hline \multicolumn{4}{|c|}{ Crozet Island } \\
\hline April 3,1973 & $47^{\circ} 7^{\prime} S$ & $49^{\circ} 4^{\prime} \mathrm{S}$ & $0.098 \pm 0.009$ \\
\hline April 4,1973 & $49^{\circ} 4^{\prime} \mathrm{S}$ & & $<0.020$ \\
\hline April 7, 1973 & \multirow{2}{*}{\multicolumn{2}{|c|}{ Kerguelen Island }} & $0.420 \pm 0.022$ \\
\hline April 8,1973 & & & $0.092 \pm 0.008$ \\
\hline April 9,1973 & $49^{\circ} 9^{\prime} \mathrm{S}$ & $48^{\circ} 8^{\prime} \mathrm{S}$ & $0.071 \pm 0.007$ \\
\hline April 10, 1973 & $48^{\circ} 9^{\prime} \mathrm{S}$ & $47^{\circ} 5^{\prime} \mathrm{S}$ & $0.025 \pm 0.006$ \\
\hline April 11, 1973 & $47^{\circ} 5^{\prime} \mathrm{S}$ & $46^{\circ} 20^{\prime} \mathrm{S}$ & 0.020 \\
\hline \multicolumn{4}{|c|}{ Crozet Island } \\
\hline April 13, 1973 & $46^{\circ} 20^{\prime} \mathrm{S}$ & $44^{\circ} 6^{\prime} S$ & $0.084 \pm 0.009$ \\
\hline April 14, 1973 & $44^{\circ} 6^{\prime} \mathrm{S}$ & $42^{\circ} 0^{\prime} \mathrm{S}$ & $0.055 \pm 0.008$ \\
\hline April 15, 1973 & $41^{\circ} 9^{\prime} \mathrm{S}$ & $39^{\circ} 2^{\prime} \mathrm{S}$ & $0.020 \pm 0.007$ \\
\hline April 16, 1973 & $39^{\circ} 2^{\prime} \mathrm{S}$ & $37^{\circ} 5^{\prime} \mathrm{S}$ & 0.020 \\
\hline \multicolumn{4}{|c|}{ Amsterdam Island } \\
\hline April 20, 1973 & $37^{\circ} 0^{\prime} \mathrm{S}$ & $32^{\circ} 9^{\prime} \mathrm{S}$ & $0.030 \pm 0.005$ \\
\hline April 21, 1973 & $32^{\circ} 9^{\prime} \mathrm{S}$ & $27^{\circ} 4^{\prime} \mathrm{S}$ & $0.025 \pm 0.006$ \\
\hline April 22, 1973 & $27^{\circ} 1^{\prime} S$ & $22^{\circ} 6^{\prime} \mathrm{S}$ & $0.040 \pm 0.004$ \\
\hline
\end{tabular}

the coast. As this is close to the background measured in the sub-Antarctic area at several thousand kilometers from continents, the continental $\mathrm{SO}_{2}$ component transported through the lower troposphere may be considered negligible.

However, the residence time of $\mathrm{SO}_{2}$ in the high remote troposphere could be rather higher than 1.5 days [Rodhe and Isaksen, 1980], and even 8 days [Logan et al., 1979], if heterogeneous reactions are neglected. Consequently, if some $\mathrm{SO}_{2}$ is

TABLE 1b. Atmospheric $\mathrm{SO}_{2}$ Concentrations Cruise $\mathrm{B}$

\begin{tabular}{cccc}
\hline & \multicolumn{2}{c}{ Latitude } & \\
\cline { 2 - 3 } Date & Start & End & $\mathrm{SO}_{2}, \mu \mathrm{g} / \mathrm{m}^{3}$ \\
\hline April 23, 1973 & $18^{\circ} 20^{\prime} \mathbf{N}$ & $20^{\circ} 40^{\prime} \mathbf{N}$ & $0.105 \pm 0.010$ \\
April 24, 1973 & $20^{\circ} 40^{\prime} \mathbf{N}$ & $23^{\circ} 05^{\prime} \mathbf{N}$ & $0.140 \pm 0.010$ \\
April 25, 1973 & $23^{\circ} 05^{\prime} \mathbf{N}$ & $25^{\circ} 57^{\prime} \mathbf{N}$ & $0.555 \pm 0.060$ \\
April 26, 1973 & $25^{\circ} 57^{\prime} \mathbf{N}$ & $29^{\circ} 08^{\prime} \mathbf{N}$ & $0.405 \pm 0.020$ \\
April 27, 1973 & $29^{\circ} 08^{\prime} \mathbf{N}$ & $31^{\circ} 56^{\prime} \mathbf{N}$ & $0.304 \pm 0.015$ \\
April 28, 1973 & $31^{\circ} 56^{\prime} \mathbf{N}$ & $34^{\circ} 28^{\prime} \mathbf{N}$ & $0.248 \pm 0.015$ \\
April 29, 1973 & $34^{\circ} 28^{\prime} \mathbf{N}$ & $36^{\circ} 40^{\prime} \mathbf{N}$ & $0.121 \pm 0.013$ \\
April 30, 1972 & $36^{\circ} 40^{\prime} \mathbf{N}$ & $38^{\circ} 55^{\prime} \mathbf{N}$ & $0.158 \pm 0.016$ \\
May 1, 1973 & $38^{\circ} 55^{\prime} \mathbf{N}$ & $40^{\circ} 35^{\prime} \mathbf{N}$ & \\
May 2, 1973 & $40^{\circ} 38^{\prime} \mathbf{N}$ & $42^{\circ} 28^{\prime} \mathbf{N}$ & $0.276 \pm 0.015$ \\
May 3, 1973 & $42^{\circ} 28^{\prime} \mathbf{N}$ & $44^{\circ} 24^{\prime} \mathbf{N}$ & $0.212 \pm 0.012$ \\
May 4, 1973 & $44^{\circ} 24^{\prime} \mathbf{N}$ & $45^{\circ} 48^{\prime} \mathbf{N}$ & $0.240 \pm 0.015$ \\
\hline
\end{tabular}

\begin{tabular}{|c|c|c|c|}
\hline \multirow[b]{2}{*}{ Date } & \multicolumn{2}{|c|}{ Latitude } & \multirow[b]{2}{*}{$\mathrm{SO}_{2} \mu \mathrm{g} / \mathrm{m}^{3}$} \\
\hline & Start & End & \\
\hline \multicolumn{4}{|c|}{ Reunion } \\
\hline April 29, 1973 & $17^{\circ} 7^{\prime} \mathrm{S}$ & $13^{\circ} 5^{\prime} \mathrm{S}$ & $0.120 \pm 0.008$ \\
\hline April 30, 1973 & $13^{\circ} 5^{\prime} S$ & $8^{\circ} 9 S$ & $0.033 \pm 0.006$ \\
\hline May 1,1973 & $8^{\circ} 9^{\prime} \mathrm{S}$ & $5^{\circ} 6^{\prime} \mathrm{S}$ & $0.100 \pm 0.010$ \\
\hline May 2,1973 & $5^{\circ} 6^{\prime} \mathrm{S}$ & $6^{\circ} 7^{\prime} \mathrm{S}$ & $0.058 \pm 0.008$ \\
\hline May 3,1973 & $6^{\circ} 7^{\prime} \mathrm{S}$ & $9^{\circ} 1^{\prime} \mathrm{S}$ & $<0.020$ \\
\hline May 4,1973 & $9^{\circ} 1^{\prime} \mathbf{S}$ & $11^{\circ} 0^{\prime} \mathrm{S}$ & $0.035 \pm 0.015$ \\
\hline May 5,1973 & $11^{\circ} 0^{\prime} \mathrm{S}$ & $14^{\circ} 9^{\prime} \mathrm{S}$ & $\ll 0.020$ \\
\hline May 6,1973 & $15^{\circ} 2^{\prime} \mathrm{S}$ & $20^{\circ} 0^{\prime} \mathrm{S}$ & $0.080 \pm 0.007$ \\
\hline May 7,1973 & $20^{\circ} \mathrm{S}$ & $23^{\circ} 4^{\prime} S$ & $0.095 \pm 0.007$ \\
\hline May 8,1973 & $23^{\circ} 4^{\prime} \mathrm{S}$ & $26^{\circ} 6^{\prime} \mathrm{S}$ & $0.052 \pm 0.006$ \\
\hline May 9,1973 & $27^{\circ} 4^{\prime} \mathrm{S}$ & $28^{\circ} 8^{\prime} \mathrm{S}$ & $0.040 \pm 0.007$ \\
\hline May 10,1973 & $28^{\circ} 8^{\prime} \mathrm{S}$ & $32^{\circ} 9^{\prime} \mathrm{S}$ & $0.148 \pm 0.008$ \\
\hline May 11,1973 & $32^{\circ} 9^{\prime} \mathrm{S}$ & $33^{\circ} 6^{\prime} \mathrm{S}$ & \\
\hline May 12,1973 & $33^{\circ} 6^{\prime} \mathrm{S}$ & $33^{\circ} 7^{\prime} \mathrm{S}$ & $0.200 \pm 0.010$ \\
\hline May 13,1973 & $33^{\circ} 8^{\prime} \mathrm{S}$ & $38^{\circ} 0^{\prime} \mathrm{S}$ & $0.110 \pm 0.007$ \\
\hline May 14,1973 & $38^{\circ} 0^{\prime} S$ & $40^{\circ} 0^{\prime} \mathrm{S}$ & $0.174 \pm 0.012$ \\
\hline May 15,1973 & $40^{\circ} 0^{\prime} \mathrm{S}$ & $43^{\circ} 6^{\prime} \mathrm{S}$ & $0.030 \pm 0.007$ \\
\hline May 16,1973 & $43^{\circ} 6^{\prime} \mathrm{S}$ & $45^{\circ} 4^{\prime} \mathrm{S}$ & $0.110 \pm 0.010$ \\
\hline May 17,1973 & $45^{\circ} 4^{\prime} \mathrm{S}$ & $47^{\circ} 0^{\prime} \mathrm{S}$ & $0.034 \pm 0.006$ \\
\hline May 18,1973 & $47^{\circ} 0^{\prime} S$ & $49^{\circ} 75^{\prime} \mathrm{S}$ & $0.065 \pm 0.007$ \\
\hline May 19,1973 & $49^{\circ} 7^{\prime} \mathrm{S}$ & $49^{\circ} 7^{\prime} S$ & $0.084 \pm 0.008$ \\
\hline \multicolumn{4}{|c|}{ Kerguelen Island } \\
\hline May 21,1973 & $49^{\circ} 7^{\prime} \mathrm{S}$ & $47^{\circ} 1^{\prime} \mathrm{S}$ & $0.140 \pm 0.013$ \\
\hline May 22,1973 & $46^{\circ} 9^{\prime} \mathrm{S}$ & $46^{\circ} 6^{\prime} S$ & $0.043 \pm 0.006$ \\
\hline May 23,1973 & $46^{\circ} 6^{\prime} \mathrm{S}$ & $43^{\circ} 7^{\prime} \mathrm{S}$ & $<0.020$ \\
\hline May 24,1973 & $43^{\circ} 7^{\prime} \mathrm{S}$ & $42^{\circ} 3^{\prime} \mathrm{S}$ & $0.088 \pm 0.009$ \\
\hline May 25,1973 & $42^{\circ} 3^{\prime} \mathrm{S}$ & $41^{\circ} 1^{\prime} \mathrm{S}$ & $0.081 \pm 0.012$ \\
\hline May 26,1973 & $38^{\circ} 9^{\prime} \mathrm{S}$ & $34^{\circ} 3^{\prime} \mathrm{S}$ & $0.062 \pm 0.008$ \\
\hline May 27,1973 & $34^{\circ} 0^{\prime} \mathrm{S}$ & $31^{\circ} 9^{\prime} \mathrm{S}$ & $<0.020$ \\
\hline May 28,1973 & $30^{\circ} 1^{\prime} \mathrm{S}$ & $29^{\circ} 5^{\prime} \mathrm{S}$ & $<0.020$ \\
\hline May 29,1973 & $28^{\circ} 9^{\prime} \mathrm{S}$ & $26^{\circ} 4^{\prime} \mathrm{S}$ & $0.025 \pm 0.004$ \\
\hline \multicolumn{4}{|c|}{ La Reunion } \\
\hline May 30,1973 & $26^{\circ} 4^{\prime} \mathrm{S}$ & $20^{\circ} 8^{\prime} \mathrm{S}$ & $0.020 \pm 0.007$ \\
\hline June 1,1973 & $20^{\circ} 8^{\prime} \mathrm{S}$ & $20^{\circ} 5^{\prime} \mathrm{S}$ & $0.197 \pm 0.012$ \\
\hline June 2,1973 & $20^{\circ} 5^{\prime} \mathrm{S}$ & $20^{\circ} 0^{\prime} \mathrm{S}$ & $0.258 \pm 0.013$ \\
\hline June 3,1973 & $20^{\circ} 0^{\prime} \mathrm{S}$ & $17^{\circ} 8^{\prime} \mathrm{S}$ & $0.072 \pm 0.008$ \\
\hline June 4,1973 & $17^{\circ} 7^{\prime} \mathrm{S}$ & $26^{\circ} \mathrm{S}$ & $0.020 \pm 0.007$ \\
\hline
\end{tabular}

carried far from the continents after its advection in the upper troposphere, it should have the same behavior as radioactive gaseous continental tracers such as ${ }^{222} \mathrm{Rn}$. In that hypothesis the residence time of $\mathrm{SO}_{2}$ would be still shorter than the residence time of ${ }^{222} \mathrm{Rn}$ ( 5.5 days). As the typical radon con-

TABLE 1d. Atmospheric $\mathrm{SO}_{2}$ Concentrations: Cruise D

\begin{tabular}{|c|c|c|c|}
\hline \multirow[b]{2}{*}{ Date } & \multicolumn{2}{|c|}{ Latitude } & \multirow[b]{2}{*}{$\mathrm{SO}_{2}, \mu \mathrm{g} / \mathrm{m}^{3}$} \\
\hline & Start & End & \\
\hline April 25,1974 & $18^{\circ} 9 \mathrm{~N}$ & $24^{\circ} 0^{\prime} \mathrm{N}$ & $0.037 \pm 0.006$ \\
\hline April 28,1974 & $24^{\circ} \mathrm{S}$ & $24^{\circ} 0^{\prime} \mathrm{N}$ & $0.036 \pm 0.016$ \\
\hline April 29,1974 & $25^{\circ} 2 \mathrm{~N}$ & $26^{\circ} 0^{\prime} \mathrm{N}$ & $0,026 \pm 0.006$ \\
\hline May 1,1974 & $26^{\circ} 0^{\prime} \mathrm{N}$ & $27^{\circ} 9^{\prime} \mathrm{N}$ & $0.072 \pm 0.008$ \\
\hline May 4,1974 & $28^{\circ} 0^{\prime} \mathrm{N}$ & $28^{\circ} 3^{\prime} \mathrm{N}$ & $0.519 \pm 0.055$ \\
\hline May 5,1974 & $28^{\circ} 3^{\prime} \mathrm{N}$ & $28^{\circ} 9^{\prime} \mathrm{N}$ & $0.224 \pm 0.015$ \\
\hline May 6,1974 & $28^{\circ} 8^{\prime} \mathrm{N}$ & $28^{\circ} 6^{\prime} \mathrm{N}$ & $0.079 \pm 0.007$ \\
\hline May 7,1974 & $28^{\circ} 6^{\prime} \mathrm{N}$ & $28^{\circ} 6^{\prime} \mathrm{N}$ & $0.086 \pm 0.008$ \\
\hline May 9,1974 & $28^{\circ} 6^{\prime} \mathrm{N}$ & $28^{\circ} 6^{\prime} \mathrm{N}$ & $0.124 \pm 0.010$ \\
\hline May 10,1974 & $28^{\circ} 6^{\prime} \mathrm{N}$ & $28^{\circ} 6^{\prime} \mathrm{N}$ & $0.980 \pm 0.065$ \\
\hline May 11,1974 & $28^{\circ} 6^{\prime} \mathrm{N}$ & $28^{\circ} 0^{\prime} \mathrm{N}$ & $0.068 \pm 0.006$ \\
\hline May 13,1974 & $28^{\circ} 0^{\prime} \mathrm{N}$ & $31^{\circ} 1^{\prime} \mathrm{N}$ & $0.299 \pm 0.015$ \\
\hline May 14,1974 & $31^{\circ} 1^{\prime} \mathrm{N}$ & $34^{\circ} 2^{\prime} \mathrm{N}$ & $0.636 \pm 0.050$ \\
\hline May 15,1974 & $34^{\circ} 2^{\prime} \mathrm{N}$ & $34^{\circ} 6^{\prime} \mathrm{N}$ & $0.087 \pm 0.009$ \\
\hline May 16,1974 & $34^{\circ} 6^{\prime} \mathrm{N}$ & $36^{\circ} 9^{\prime} \mathrm{N}$ & $0.076+0.007$ \\
\hline
\end{tabular}


TABLE 1e. Atmospheric $\mathrm{SO}_{2}$ Concentrations: Cruise $\mathrm{E}$

\begin{tabular}{|c|c|c|c|}
\hline \multirow[b]{2}{*}{ Date } & \multicolumn{2}{|c|}{ Latitude } & \multirow[b]{2}{*}{$\mathrm{SO}_{2}, \mu \mathrm{g} / \mathrm{m}^{3}$} \\
\hline & Start & End & \\
\hline May 22, 1974 & $37^{\circ} 0^{\prime} \mathrm{N}$ & $35^{\circ} 6^{\prime} \mathrm{N}$ & $0.208 \pm 0.014$ \\
\hline May 26,1974 & $36^{\circ} 8^{\prime} \mathrm{N}$ & $36^{\circ} 0^{\mathrm{N}}$ & $0.260 \pm 0.015$ \\
\hline May 30,1974 & $36^{\circ} 9^{\prime} \mathrm{N}$ & $36^{\circ} 6^{\prime} \mathrm{N}$ & $0.947 \pm 0.070$ \\
\hline May 31,1974 & $37^{\circ} 0^{\prime} \mathrm{N}$ & $36^{\circ} 9^{\prime} \mathbf{N}$ & $0.314 \pm 0.016$ \\
\hline June 1,1974 & $37^{\circ} 0^{\circ} \mathrm{N}$ & $36^{\circ} 9^{\prime} \mathrm{N}$ & $0.157 \pm 0.013$ \\
\hline June 2,1974 & $37^{\circ} 0^{\prime} \mathrm{N}$ & $35^{\circ} 7^{\prime} \mathrm{N}$ & $0.059 \pm 0.006$ \\
\hline June 3,1974 & $35^{\circ} 7 \mathrm{~N}$ & $34^{\circ} 3^{\prime} \mathrm{N}$ & $0.126 \pm 0.011$ \\
\hline June 4,1974 & $34^{\circ} 3^{\prime} \mathrm{N}$ & $32^{\circ} 9^{\prime} \mathrm{N}$ & $0.157 \pm 0.012$ \\
\hline June 8,1974 & $28^{\circ} 6^{\prime} \mathrm{N}$ & $28^{\circ} 6^{\prime} \mathrm{N}$ & $0.594 \pm 0.055$ \\
\hline June 9,1974 & $28.6^{\prime} \mathrm{N}$ & $23.7^{\prime} \mathrm{N}$ & $0.254 \pm 0.014$ \\
\hline June 10,1974 & $23^{\circ} 7^{\prime} \mathrm{N}$ & $20^{\circ} 2^{\prime} \mathrm{N}$ & $0.528 \pm 0.040$ \\
\hline June 11,1974 & $20^{\circ} 2^{\prime} \mathrm{N}$ & $15^{\circ} 9^{\prime} \mathrm{N}$ & $0.745 \pm 0.060$ \\
\hline
\end{tabular}

centrations obtained during these three expeditions in the remote marine areas is generally less than $2 \mathrm{pCi} / \mathrm{m}^{3}$ (100 to 1000 times less than the continental values), $\mathrm{SO}_{2}$ from continental origin would not normally contribute to the $\mathrm{SO}_{2}$ levels measured in the boundary layer of the sub-Antarctic marine atmosphere. For this region, another source is required.

Could the $\mathrm{SO}_{2}$ be produced above the open ocean by the oxidation of gaseous sulfur compounds with high residence times (several months), such as $C S_{2}$ and $\operatorname{COS}$ ? COS may be produced by the oxidation of $\mathrm{CS}_{2}$ and may be a precursor of $\mathrm{SO}_{2}$ via the oxidation with HO radical [Crutzen, 1976; Kurylo, 1978a; Logan et al., 1979; Sze and Ko, 1980; Torres et al., 1980].

$$
\begin{aligned}
& \mathrm{COS}+\mathrm{HO} \rightarrow \mathrm{CO}_{2}+\mathrm{SH}^{\cdot} \\
& \mathrm{CS}_{2}+\mathrm{HO} \rightarrow \mathrm{COS}+\mathrm{SH}^{\circ}
\end{aligned}
$$

In the atmosphere, $\mathrm{SH} \cdot$ radicals probably oxidize into $\mathrm{SO}_{2}$ by the following principal reactions [Perry et al., 1976; $\mathrm{McEl}$ roy et al., 1980]:

\begin{tabular}{|c|c|c|c|}
\hline \multirow[b]{2}{*}{ Date } & \multicolumn{2}{|c|}{ Longitude } & \multirow[b]{2}{*}{$\mathrm{SO}_{2}, \mu \mathrm{g} / \mathrm{m}^{3}$} \\
\hline & Start & End & \\
\hline June 21,1974 & $19^{\circ} 1^{\prime} \mathrm{W}$ & $22^{\circ} 3^{\prime} \mathrm{W}$ & $0.104 \pm 0.010$ \\
\hline June 22,1974 & $22^{\circ} 3^{\prime} \mathrm{W}$ & $26^{\circ} 0^{\prime} \mathrm{W}$ & $0.178 \pm 0.013$ \\
\hline June 23, 1974 & $26^{\circ} 0^{\prime} \mathrm{W}$ & $30^{\circ} 3^{\prime} \mathrm{W}$ & $0.037 \pm 0.009$ \\
\hline June 24, 1974 & $30^{\circ} 3^{\prime} \mathrm{W}$ & $34^{\circ} 2^{\prime} \mathrm{W}$ & $0.070 \pm 0.007$ \\
\hline June 25,1974 & $34^{\circ} 2^{\prime} \mathrm{W}$ & $35^{\circ} 0^{\prime} \mathrm{W}$ & $0.045 \pm 0.005$ \\
\hline June 26,1974 & $35^{\circ} 0^{\prime} \mathrm{W}$ & $35^{\circ} 0^{\prime} \mathrm{W}$ & $0.045 \pm 0.005$ \\
\hline June 27, 1974 & $35^{\circ} 0^{\prime} \mathrm{W}$ & $35^{\circ} 0^{\prime} \mathrm{W}$ & $0.030 \pm 0.006$ \\
\hline June 28,1974 & $35^{\circ} 0^{\prime} \mathrm{W}$ & $35^{\circ} 0^{\prime} \mathrm{W}$ & $0.068 \pm 0.006$ \\
\hline June 29,1974 & $35^{\circ} 0^{\prime} \mathrm{W}$ & $35^{\circ} 0^{\prime} \mathrm{W}$ & $0.090 \pm 0.008$ \\
\hline June 30,1974 & $35^{\circ} 0^{\prime} \mathrm{W}$ & $35^{\circ} 0^{\prime} \mathrm{W}$ & $0.050 \pm 0.005$ \\
\hline July 1,1974 & $35^{\circ} 0^{\prime} \mathrm{W}$ & $35^{\circ} 0^{\prime} \mathrm{W}$ & $0.118 \pm 0.010$ \\
\hline July 2,1974 & $35^{\circ} 0^{\prime} \mathrm{W}$ & $35^{\circ} 0^{\prime} \mathrm{W}$ & $0.075 \pm 0.007$ \\
\hline July 3,1974 & $35^{\circ} 0^{\prime} \mathrm{W}$ & $35^{\circ} 0^{\prime} \mathrm{W}$ & $0.137 \pm 0.011$ \\
\hline July 4,1974 & $35^{\circ} 0^{\prime} \mathrm{W}$ & $35^{\circ} 0^{\prime} \mathrm{W}$ & $0.063 \pm 0.006$ \\
\hline July 5,1974 & $35^{\circ} 0 \mathrm{~W}$ & $35^{\circ} 0^{\prime} \mathrm{W}$ & $0.073 \pm 0.007$ \\
\hline July 6,1974 & $35^{\circ} 0^{\prime} \mathrm{W}$ & $35^{\circ} 0^{\prime} \mathrm{W}$ & $0.030 \pm 0.005$ \\
\hline July 7,1974 & $35^{\circ} 0^{\prime} \mathrm{W}$ & $35^{\circ} 0 \mathrm{~W}$ & $0.020 \pm 0.005$ \\
\hline July 8,1974 & $35^{\circ} 0^{\prime} \mathrm{W}$ & $35^{\circ} 0^{\circ} \mathrm{W}$ & $0.020 \pm 0.005$ \\
\hline July 9,1974 & $35^{\circ} 0^{\prime} \mathrm{W}$ & $35^{\circ} 0 \mathrm{~W}$ & $0.032 \pm 0.006$ \\
\hline July 10,1974 & $35^{\circ} 0^{\prime} \mathrm{W}$ & $35^{\circ} 0^{\prime} \mathrm{W}$ & $0.040 \pm 0.006$ \\
\hline July 11,1974 & $35^{\circ} 0^{\prime} \mathrm{W}$ & $35^{\circ} 0^{\prime} \mathrm{W}$ & $0.050 \pm 0.006$ \\
\hline July 12,1974 & $35^{\circ} 0^{\prime} \mathrm{W}$ & $35^{\circ} 0^{\prime} \mathrm{W}$ & $0.040 \pm 0.006$ \\
\hline July 13,1974 & $35^{\circ} 0^{\prime} \mathrm{W}$ & $35^{\circ} 0 \mathrm{~W}$ & $0.035 \pm 0.006$ \\
\hline July 14,1974 & $35^{\circ} 0^{\prime} \mathrm{W}$ & $32^{\circ} 9^{\prime} \mathrm{W}$ & $0.036 \pm 0.007$ \\
\hline July 15,1974 & $32^{\circ} 9^{\prime} \mathrm{W}$ & $28^{\circ} 5^{\prime} \mathrm{W}$ & $0.020 \pm 0.005$ \\
\hline July 16,1974 & $28^{\circ} 5^{\prime} \mathrm{W}$ & $22^{\circ} 7^{\prime} W$ & $0.022 \pm 0.005$ \\
\hline
\end{tabular}

TABLE 1 f. Atmospheric $\mathrm{SO}_{2}$ Concentrations: Cruise $\mathrm{F}$

\begin{tabular}{|c|c|c|c|}
\hline \multirow[b]{2}{*}{ Date } & \multicolumn{2}{|c|}{ Latitude } & \multirow[b]{2}{*}{$\mathrm{SO}_{2}, \mu \mathrm{g} / \mathrm{m}^{3}$} \\
\hline & Start & End & \\
\hline January 16,1974 & $24.1^{\circ} \mathrm{S}$ & $28.0^{\circ} \mathrm{S}$ & $0.0 \pm 0.005$ \\
\hline January 17,1974 & $28.0^{\circ} \mathrm{S}$ & $32.0^{\circ} \mathrm{S}$ & $0.032 \pm 0.005$ \\
\hline January 18,1974 & $32.0^{\circ} \mathrm{S}$ & $36.2^{\circ} \mathrm{S}$ & $0.067 \pm 0.006$ \\
\hline January 19,1974 & $36.2^{\circ} \mathrm{S}$ & Amsterdam & $0.142 \pm 0.011$ \\
\hline January 21,1974 & \multicolumn{2}{|c|}{ Amsterdam Island } & $0.172 \pm 0.014$ \\
\hline January 22,1974 & Amsterdam & $39.2^{\circ} \mathrm{S}$ & $0.074 \pm 0.007$ \\
\hline January 23,1974 & $39.2^{\circ} \mathrm{S}$ & $40.9^{\circ} \mathrm{S}$ & $0.080 \pm 0.007$ \\
\hline January 24,1974 & $40.9^{\circ} \mathrm{S}$ & $43.0^{\circ} \mathrm{S}$ & $0.085 \pm 0.007$ \\
\hline January 26,1974 & $45.1^{\circ} \mathrm{S}$ & Crozet & $0.044 \pm 0.006$ \\
\hline January 27,1974 & \multicolumn{2}{|c|}{ Crozet Island } & $0.093 \pm 0.009$ \\
\hline January 28,1974 & \multicolumn{2}{|c|}{ Crozet Island } & $0.095 \pm 0.009$ \\
\hline January 29, 1974 & Crozet & $47.3^{\circ} \mathrm{S}$ & $0.484 \pm 0.035$ \\
\hline January 30,1974 & $47.4^{\circ} \mathrm{S}$ & $49.0^{\circ} \mathrm{S}$ & $0.709 \pm 0.060$ \\
\hline January 31, 1974 & $49.0^{\circ} \mathrm{S}$ & Kerguelen & $0.687 \pm 0.060$ \\
\hline February 2,1974 & \multicolumn{2}{|c|}{ Kerguelen Island } & $0.068 \pm 0.007$ \\
\hline February 3,1974 & Kerguelen & $46.9^{\circ} \mathrm{S}$ & $0.203 \pm 0.012$ \\
\hline February 4,1974 & $46.9^{\circ} \mathrm{S}$ & $40.4^{\circ} \mathrm{S}$ & $0.036 \pm 0.005$ \\
\hline 6,1974 & $37.8^{\circ} \mathrm{S}$ & $35.2^{\circ} \mathrm{S}$ & \pm 0.006 \\
\hline February 7, 1974 & $35.2^{\circ} \mathrm{S}$ & $31.0^{\circ} \mathrm{S}$ & $0.245 \pm 0.014$ \\
\hline November 26,1974 & Marseille & $41^{\circ} 9^{\prime} \mathrm{N}$ & $0.077 \pm 0.007$ \\
\hline November 27,1974 & $41^{\circ} 9^{\prime} \mathrm{N}$ & $37^{\circ} 3^{\prime} \mathrm{N}$ & $0.188 \pm 0.009$ \\
\hline November 28,1974 & $37^{\circ} 3^{\prime} \mathrm{N}$ & $34^{\circ} 4^{\prime} \mathrm{N}$ & $0.071 \pm 0.007$ \\
\hline November 29,1974 & $34^{\circ} 4^{\prime} \mathrm{N}$ & $29^{\circ} \mathrm{N}$ & $0.071 \pm 0.007$ \\
\hline aber 30,1974 & $29^{\circ} 6^{\prime} \mathrm{N}$ & $24^{\circ} 4^{\prime} \mathrm{N}$ & $0.068 \pm 0.007$ \\
\hline aber 1,1974 & $24^{\circ} 4^{\prime} \mathrm{N}$ & $18^{\circ} 2^{\prime} \mathrm{N}$ & $2 \pm 0.009$ \\
\hline nber 2,1974 & $18^{\circ} 2^{\prime} \mathrm{N}$ & $12^{\circ} 7^{\prime} \mathrm{N}$ & $0.066 \pm 0.006$ \\
\hline December 3, 1974 & $12^{\circ} 7^{\prime} \mathrm{N}$ & $7^{\circ} 5^{\prime} \mathrm{N}$ & $0.094 \pm 0.009$ \\
\hline ber 4,1974 & $7^{\circ} 5^{\prime} \mathrm{N}$ & $2^{\circ} 9^{\prime} \mathrm{N}$ & $0.086 \pm 0.008$ \\
\hline ber 5,1974 & $2^{\circ} 9^{\prime} \mathrm{N}$ & $1^{\circ} 7^{\prime} \mathrm{S}$ & \pm 0.006 \\
\hline ber 6,1974 & $1^{\circ} 7^{\prime} \mathrm{S}$ & $6^{\circ} 6^{\prime} \mathrm{S}$ & $0.041 \pm 0.008$ \\
\hline ber 7,1974 & $6^{\circ} 6^{\prime} S$ & $11^{\circ} 4^{\prime} \mathrm{S}$ & $0.055+0.006$ \\
\hline ber 8,1974 & $11^{\circ} 4^{\prime} \mathrm{S}$ & $16^{\circ}$ & $9 \pm 0.006$ \\
\hline er 9,1974 & $16^{\circ} 3^{\prime} \mathrm{S}$ & $21^{\circ} 2^{\prime} \mathrm{S}$ & $0.038 \pm 0.006$ \\
\hline December 10,1974 & $21^{\circ} 2^{\prime} \mathrm{S}$ & $25^{\circ} 6^{\prime} \mathrm{S}$ & $0.043 \pm 0.006$ \\
\hline December 11,1974 & & & $0.149 \pm 0.011$ \\
\hline er 12,1974 & $29^{\circ} 1^{\prime} \mathrm{S}$ & 33 & $7 \pm 0.008$ \\
\hline er 15,1974 & $36^{\circ} 8^{\prime} \mathrm{S}$ & $42^{\circ} 3^{\prime} \mathrm{S}$ & $0.017 \pm 0.005$ \\
\hline December 15, 1974 & $39^{\circ} 1^{\prime} \mathrm{S}$ & $43^{\circ} 7^{\prime} \mathrm{S}$ & $0.048 \pm 0.008$ \\
\hline December 17,1974 & $45^{\circ} 6^{\prime} \mathrm{S}$ & $46^{\circ} 9^{\prime} S$ & $0.170 \pm 0.012$ \\
\hline lber 19, 1974 & $45^{\circ} 7^{\prime} \mathrm{S}$ & $48^{\circ} 2 ' S$ & $0.149 \pm 0.011$ \\
\hline ber 19,1974 & $46^{\circ} 0^{\prime} \mathrm{S}$ & $49^{\circ} 0^{\prime} \mathrm{S}$ & $0.159 \pm 0.011$ \\
\hline January 5,1975 & $46^{\circ} 5^{\prime} \mathrm{S}$ & $42^{\circ} 3^{\prime} \mathrm{S}$ & $0.013 \pm 0.004$ \\
\hline January 6,1974 & $42^{\circ} 3^{\prime} \mathrm{S}$ & $38^{\circ} 0^{\prime} S$ & $0.014 \pm 0.004$ \\
\hline January 7,1974 & $38^{\circ} \mathrm{S}$ & $32^{\circ} 2^{\prime} \mathrm{S}$ & $0.169 \pm 0.012$ \\
\hline January 8,1975 & $32^{\circ} 2^{\prime} \mathrm{S}$ & $26^{\circ} 3^{\prime} \mathrm{S}$ & $0.019 \pm 0.005$ \\
\hline January 9,1975 & $26^{\circ} 3^{\prime} \mathrm{S}$ & La Reunion & $0.040 \pm 0.006$ \\
\hline January 15,1975 & a Reunion & $24^{\circ} 1^{\prime} S$ & $0.042 \pm 0.006$ \\
\hline
\end{tabular}

TABLE 1g. Atmospheric $\mathrm{SO}_{2}$ Concentrations: Cruise $\mathrm{G}$

$$
\begin{aligned}
& \mathrm{SH}^{\cdot}+\mathrm{O}_{2} \rightarrow \mathrm{SO}^{\cdot}+\mathrm{OH}^{-} \\
& \mathrm{SO}^{+}+\mathrm{O}_{2} \rightarrow \mathrm{SO}_{2}+\mathrm{O}^{\prime}
\end{aligned}
$$

A great discrepancy still exists regarding the kinetic constant $k_{1}$ of (1); the advanced values range between $5.7 \times 10^{-14}$ $\mathrm{cm}^{3}$ molecule ${ }^{-1} \mathrm{~s}^{-1}$ [Kurylo, 1978a] and $8.8 \times 10^{-15} \mathrm{~cm}^{3}$ molecule ${ }^{-1} \mathrm{~s}^{-1}$ [Ravishankara et al., 1980].

A larger uncertainty exists for the kinetic constant of (2); $k_{2}$ varies between $4.3 \times 10^{-12} \mathrm{~cm}^{3}$ molecule ${ }^{-1} \mathrm{~s}^{-1}$ and $1.5 \times$ $10^{-15} \mathrm{~cm}^{3}$ molecule ${ }^{-1} \mathrm{~s}^{-1}$ [Kurylo, 1978a; Wine et al., 1980].

In any case the oxidation rate in (2) is faster than the oxidation rate in (1), the rate production of $\mathrm{SO}_{2}$ from these sulfur compounds essentially depends on (1). Chameides and Tan [1981] have calculated concentrations increasing from 0.5 $\times 10^{5}$ radicals $\mathrm{cm}^{-3}$ in high latitudes to $2.5 \times 10^{6}$ radicals $\mathrm{cm}^{-3}$ in equatorial and tropical areas. Moreover, Logan et al. [1979] have computed $\mathrm{OH}$ profiles where the maximum concentrations for the low troposphere was $3 \times 10^{6}$ radicals 
TABLE 1h. Atmospheric $\mathrm{SO}_{2}$ Concentrations: Cruise $\mathrm{H}$

\begin{tabular}{|c|c|c|c|}
\hline \multirow[b]{2}{*}{ Date } & \multicolumn{2}{|c|}{ Latitude } & \multirow[b]{2}{*}{$\mathrm{SO}_{2}, \mu \mathrm{g} / \mathrm{m}^{3}$} \\
\hline & Start & End & \\
\hline February 22, 1977 & $21.6^{\circ} \mathrm{S}$ & $23.2^{\circ} \mathrm{S}$ & $0.129 \pm 0.011$ \\
\hline February 23,1977 & $24.0^{\circ} \mathrm{S}$ & $25.0^{\circ} \mathrm{S}$ & $0.054 \pm 0.005$ \\
\hline February 23, 1977 & $25.0^{\circ} \mathrm{S}$ & $26.9^{\circ} \mathrm{S}$ & $0.043 \pm 0.004$ \\
\hline February 24,1977 & $27.5^{\circ} \mathrm{S}$ & $30.4^{\circ} \mathrm{S}$ & $0.041 \pm 0.004$ \\
\hline February 25, 1977 & $30.4^{\circ} \mathrm{S}$ & $34.2^{\circ} \mathrm{S}$ & $0.010 \pm 0.003$ \\
\hline February 26,1977 & $34.4^{\circ} \mathrm{S}$ & $35.5^{\circ} \mathrm{S}$ & $0.127 \pm 0.010$ \\
\hline February 26, 1977 & $35.5^{\circ} \mathrm{S}$ & Amsterdam & $0.015 \pm 0.004$ \\
\hline February 28, 1977 & Amsterdam & $39.8^{\circ} \mathrm{S}$ & $0.058 \pm 0.005$ \\
\hline March 1, 1974 & $39.8^{\circ} \mathrm{S}$ & $41.3^{\circ} \mathrm{S}$ & $0.176 \pm 0.012$ \\
\hline March 2, 1974 & $41.3^{\circ} \mathrm{S}$ & $43.0^{\circ} \mathrm{S}$ & $0.243 \pm 0.015$ \\
\hline March 3, 1974 & $43.0^{\circ} \mathrm{S}$ & Kerguelen & $0.027 \pm 0.005$ \\
\hline March 5, 1974 & $49.0^{\circ} \mathrm{S}$ & $47.5^{\circ} \mathrm{S}$ & $0.093 \pm 0.009$ \\
\hline March 6, 1974 & $47.5^{\circ} \mathrm{S}$ & $46.7^{\circ} \mathrm{S}$ & $0.119 \pm 0.010$ \\
\hline March 6, 1974 & $46.7^{\circ} \mathrm{S}$ & $46.0^{\circ} \mathrm{S}$ & $0.226 \pm 0.015$ \\
\hline March 7, 1974 & $46.0^{\circ} \mathrm{S}$ & $46.0^{\circ} \mathrm{S}$ & $0.023 \pm 0.004$ \\
\hline March 7, 1974 & $46.0^{\circ} \mathrm{S}$ & $46.7^{\circ} \mathrm{S}$ & $0.031 \pm 0.005$ \\
\hline March 8, 1974 & $46.7^{\circ} \mathrm{S}$ & $48.3^{\circ} \mathrm{S}$ & $0.022 \pm 0.005$ \\
\hline March 8, 1974 & $48.3^{\circ} \mathrm{S}$ & $49.1^{\circ} \mathrm{S}$ & $0.020 \pm 0.004$ \\
\hline March 9, 1974 & $49.1^{\circ} \mathrm{S}$ & $49.8^{\circ} \mathrm{S}$ & $0.010 \pm 0.003$ \\
\hline March 9, 1974 & $49.8^{\circ} \mathrm{S}$ & $50.4^{\circ} \mathrm{S}$ & $0.013 \pm 0.003$ \\
\hline March 10,1974 & $51.3^{\circ} \mathrm{S}$ & $52.0^{\circ} \mathrm{S}$ & $0.033 \pm 0.005$ \\
\hline March 10,1974 & $52.0^{\circ} \mathrm{S}$ & $52.8^{\circ} \mathrm{S}$ & $<0.010$ \\
\hline March 11,1974 & $52.8^{\circ} \mathrm{S}$ & $52.8^{\circ} \mathrm{S}$ & $0.035 \pm 0.005$ \\
\hline March 11,1974 & $52.8^{\circ} \mathrm{S}$ & $53.6^{\circ} \mathrm{S}$ & $0.017 \pm 0.004$ \\
\hline March 12,1974 & $53.6^{\circ} \mathrm{S}$ & $54.4^{\circ} \mathrm{S}$ & $0.166 \pm 0.012$ \\
\hline March 12,1974 & $54.4^{\circ} \mathrm{S}$ & $56.3^{\circ} \mathrm{S}$ & $0.044 \pm 0.004$ \\
\hline March 13, 1974 & $56.3^{\circ} \mathrm{S}$ & $57.4^{\circ} \mathrm{S}$ & $0.074 \pm 0.007$ \\
\hline March 14, 1974 & $57.4^{\circ} \mathrm{S}$ & $58.4^{\circ} \mathrm{S}$ & $0.093 \pm 0.009$ \\
\hline March 14, 1974 & $58.4^{\circ} \mathrm{S}$ & $59.4^{\circ} \mathrm{S}$ & $0.056 \pm 0.005$ \\
\hline March 15, 1974 & $59.4^{\circ} \mathrm{S}$ & $60.4^{\circ} \mathrm{S}$ & $0.099 \pm 0.009$ \\
\hline March 15, 1974 & $60.4^{\circ} \mathrm{S}$ & $60.4^{\circ} \mathrm{S}$ & $0.112 \pm 0.010$ \\
\hline March 16, 1974 & $60.4^{\circ} \mathrm{S}$ & $61.2^{\circ} \mathrm{S}$ & $0.022 \pm 0.004$ \\
\hline March 16, 1974 & $61.2^{\circ} \mathrm{S}$ & $62.2^{\circ} \mathrm{S}$ & $0.030 \pm 0.005$ \\
\hline March 17, 1974 & $62.2^{\circ} \mathrm{S}$ & $62.2^{\circ} \mathrm{S}$ & $0.095 \pm 0.008$ \\
\hline March 17, 1974 & $62.2^{\circ} \mathrm{S}$ & $62.2^{\circ} \mathrm{S}$ & $0.032 \pm 0.005$ \\
\hline March 18, 1974 & $62.2^{\circ} \mathrm{S}$ & $59.2^{\circ} \mathrm{S}$ & $0.011 \pm 0.003$ \\
\hline March 19, 1974 & $59.2^{\circ} \mathrm{S}$ & $57.5^{\circ} \mathrm{S}$ & $0.101 \pm 0.010$ \\
\hline March 19, 1974 & $57.5^{\circ} \mathrm{S}$ & $55.3^{\circ} \mathrm{S}$ & $0.040 \pm 0.004$ \\
\hline March 20, 1974 & $55.3^{\circ} \mathrm{S}$ & $55.3^{\circ} \mathrm{S}$ & $0.010 \pm 0.003$ \\
\hline March 20, 1974 & $55.3^{\circ} \mathrm{S}$ & $55.4^{\circ} \mathrm{S}$ & $0.157 \pm 0.012$ \\
\hline March 21, 1974 & $55.4^{\circ} \mathrm{S}$ & $54.8^{\circ} \mathrm{S}$ & $0.014 \pm 0.003$ \\
\hline March 21, 1974 & $54.8^{\circ} \mathrm{S}$ & $52.6^{\circ} \mathrm{S}$ & $<0.010$ \\
\hline March 22, 1974 & $52.6^{\circ} \mathrm{S}$ & $50.3^{\circ} \mathrm{S}$ & $0.18 \pm 0.004$ \\
\hline March 22, 1974 & $50.3^{\circ} \mathrm{S}$ & $47.8^{\circ} \mathrm{S}$ & $0.347 \pm 0.016$ \\
\hline March 24, 1974 & $47.8^{\circ} \mathrm{S}$ & $42.9^{\circ} \mathrm{S}$ & $<0.010$ \\
\hline March 24, 1974 & $42.9^{\circ} \mathrm{S}$ & $42.9^{\circ} \mathrm{S}$ & $<0.010$ \\
\hline March 25, 1974 & $42.9^{\circ} \mathrm{S}$ & $42.8^{\circ} \mathrm{S}$ & $0.012 \pm 0.003$ \\
\hline March 25, 1974 & $42.8^{\circ} \mathrm{S}$ & $43.9^{\circ} \mathrm{S}$ & $<0.010$ \\
\hline March 26, 1974 & $43.9^{\circ} \mathrm{S}$ & $45.15^{\circ} \mathrm{S}$ & $0.116 \pm 0.011$ \\
\hline March 26, 1974 & $45.1^{\circ} \mathrm{S}$ & $45.2^{\circ} \mathrm{S}$ & $0.054 \pm 0.005$ \\
\hline March 27, 1977 & $45.2^{\circ} \mathrm{S}$ & $45.2^{\circ} \mathrm{S}$ & $0.119 \pm 0.011$ \\
\hline March 27, 1977 & $45.2^{\circ} \mathrm{S}$ & $46.0^{\circ} \mathrm{S}$ & $0.052 \pm 0.005$ \\
\hline March 31, 1977 & $46.0^{\circ} \mathrm{S}$ & $45.4^{\circ} \mathrm{S}$ & $0.020 \pm 0.004$ \\
\hline March 31, 1977 & $45.4^{\circ} \mathrm{S}$ & $45.4^{\circ} \mathrm{S}$ & $0.0 \pm 0.004$ \\
\hline April 1, 1977 & $45.4^{\circ} \mathrm{S}$ & $43.5^{\circ} \mathrm{S}$ & $0.279 \pm 0.015$ \\
\hline April 2, 1977 & $43.5^{\circ} \mathrm{S}$ & $39.5^{\circ} \mathrm{S}$ & $0.010 \pm 0.003$ \\
\hline April 3, 1977 & $39.5^{\circ} \mathrm{S}$ & $34.5^{\circ} \mathrm{S}$ & $0.010 \pm 0.003$ \\
\hline
\end{tabular}

$\mathrm{cm}^{-3}$. By using this last value, which leads to maximum $\mathrm{SO}_{2}$ production, the residence time of $\mathrm{COS}$ should comprise between 67 days and 1.2 years and the residence time of $\mathrm{CS}_{2}$ between 8 days and 7 years. Even taking into account the faster reaction rate for $k_{1}$, which corresponds to the 67-day residence time for COS; a $\operatorname{COS}$ mixing ratio of 500 pptv [Torres et al., 1980]; and a 0.7-day residence time for $\mathrm{SO}_{2}$, and by considering a steady state, we could only explain a 5 pptv of $\mathrm{SO}_{2}\left(15 \times 10^{-9} \mathrm{~g} \mathrm{~m}^{3}\right)$ produced by the oxidation of COS. Rodhe and Isaksen [1980] also found that the contri- bution of $\mathrm{COS}$ to the concentration of $\mathrm{SO}_{2}$ in the low troposphere is less than 5 pptv of $\mathrm{SO}_{2}$.

This value is much lower than the 65 pptv theoretical value coming from both $\mathrm{COS}$ and $\mathrm{CS}_{2}$ oxidation [Logan et al., 1979]. But this calculation refers to a concentration at a $6-\mathrm{km}$ altitude where the residence time of $\mathrm{SO}_{2}$ is probably larger than in the boundary layer.

An experimental determination of $\mathrm{SO}_{2}$ concentration has been performed in a particular region where no sources other than long-lived gaseous precursors may interfere.

In collaboration with the "Laboratoire de Glaciologie de Grenoble (France)," $\mathrm{SO}_{2}$ has been sampled at dome $\mathrm{C}$ in the Antarctic continent $\left(7^{\circ} 40^{\prime} \mathrm{S}, 128^{\circ} 50^{\prime} \mathrm{E}\right.$; elevation, $3200 \mathrm{~m}$; distance from the coast, $1070 \mathrm{~km}$ ). The samples have been collected with the same device used by Nguyen et al. [1974a] in marine regions, except that it was enclosed in a temperaturecontrolled box at $+20^{\circ} \mathrm{C}$.

The $\mathrm{SO}_{2}$ concentrations of the 30 samples collected were all less than our detection limit of $0.02 \mu \mathrm{g} / \mathrm{m}^{3}$.

From these data in the Antarctic area we can propose the two following alternatives: either both of the low-temperature conditions and low HO- radical concentrations lessen the $\mathrm{SO}_{2}$ production efficiency, or this process alone cannot account for more than $0.02 \mu \mathrm{g} / \mathrm{m}^{3}$, which corresponds to $20 \%$ of the background $\mathrm{SO}_{2}$ we measured above the ocean.

Could the $\mathrm{SO}_{2}$ be produced over the open ocean from oxidation of $\mathrm{H}_{2} \mathrm{~S}$ ? The occurrence of atmospheric $\mathrm{H}_{2} \mathrm{~S}$ over the open ocean could come from the transfer of this compound from sediment, where its production is important, to the sea surface. This hypothesis is unlikely because the half-life of $\mathbf{H}_{2} \mathrm{~S}$ in seawater is on the order of several minutes [Ostlund and Alexander, 1963].

$\mathrm{H}_{2} \mathrm{~S}$ could also be carried from coastal areas or be locally produced either from a biogenic process at the sea surface or by the recombination of radicals issued from the oxidation of $\mathrm{COS}$ and $\mathrm{CS}_{2}$ [McElroy, 1980].

For instance, $\mathrm{H}_{2} \mathrm{~S}$ has been observed in the air by Delmas and Servant [1982], who found $13 \mathrm{pptv}$, at a 500-km distance from the coast, in the Guinea Gulf. Moreover, Slatt et al. [1978] measured concentrations ranging between 5 and 50 pptv at the coast of Florida, at Barbados, and at Sal Islands. However, these islands may not be considered as representative of open-ocean conditions, since a possible local $\mathrm{H}_{2} \mathrm{~S}$ production may occur along the littoral by the organic matter degradation. Furthermore, an atmospheric residence time for $\mathrm{H}_{2} \mathrm{~S}$, ranging between 6 and 24 hours, has been determined by Cox [1975]. Unfortunately, no data is available in remote sub-Antarctic open ocean, but if concentrations comparable to those found by Delmas et al. [1980] occur in such remote areas, $\mathrm{H}_{2} \mathrm{~S}$ may be considered as a possible precursor of $\mathrm{SO}_{2}$.

Could the $\mathrm{SO}_{2}$ be produced above the ocean by the oxidation of reactive sulfur compounds other than $\mathrm{H}_{2} \mathrm{~S}$ ? Other sulfur compounds already have been observed as well in seawater as in the marine atmosphere and could also be possible precursors of this $\mathrm{SO}_{2}$ residual concentration. Considering this hypothesis by using known results and new data, we obtained

1. The marine organic sulfides: (a) In seawater. Armstrong and Boalch [1960] have noted the occurrence of organic sulfides in seawater which, although unidentified, could include dimethylsulfide. According to Schiff [1962], the production of dimethylsulfide by various marine algae (Polysiphonia lanosa) may be due to an enzymatic action such as degradation of dimethyl propiothetin: 
<smiles>C[S+](C)CCC(=O)[O-]</smiles>

Furthermore, the degradation of methionine leads to methanethiol. Rasmussen [1974] has observed organic sulfides such as dimethylsulfide, diethyl disulfide, and methanethiol emitted by laboratory cultures of various marine algae. Andreae [1980] has presented a scheme for the marine cycle of sulfur compounds of biological origin which relates DMS and dimethylsulfoxide (DMSO) production to phytoplankton activity. Lovelock et al. [1972] have observed a mean concentration of DMS in the surface water of the Atlantic Ocean of about $12 \times 10^{-9} \mathrm{~g} / 1$. Nguyen et al. [1978a,b] have reported DMS concentrations from 11 to $580 \times 10^{-9} \mathrm{~g} / 1$ in surface waters of the Atlantic and Indian oceans and the Mediterranean Sea. More recent data obtained in the surface water of the Pacific Ocean during an oceanographic cruise from Panama to Hobart (Tasmania) show higher DMS concentrations reaching $1150 \times 10^{-9} \mathrm{~g} / \mathrm{l}$ (B. C. Nguyen et al., unpublished manuscript, 1983). Likewise, Barnard et al. [1982] have observed DMS concentrations in the surface seawater of the Atlantic Ocean that range from 34 to $1440 \times 10^{-9} \mathrm{~g} / \mathrm{l}$ with a mean value of $176 \times 10^{-9} \mathrm{~g} / \mathrm{l}$.

Depth distributions of DMS often show maxima within the upper 50 meters in the euphotic zone [Barnard et al., 1982] associated with boundary water masses of different densities [Nguyen et al., 1978a]. Also, it seems that DMS does not only originate from phytoplankton activity because high concentrations may be observed at depths as great as $2000 \mathrm{~m}$ (B. C. Nguyen et al., unpublished manuscript, 1983).

(b) In marine atmosphere. The dimethylsulfide produced in seawater may be released to the atmosphere from the sea surface because of its low solubility. Maroulis and Bandy [1977] have determined DMS concentrations of 0 to 62 pptv ( 0 to $170 \times 10^{-9} \mathrm{~g} / \mathrm{m}^{3}$ ) by cryogenic sampling in a littoral zone. Barnard et al. [1982] found 4 to $88 \times 10^{-9} \mathrm{~g} \mathrm{~m}^{-3}$ of DMS above the Atlantic Ocean. More recently we found, above the Pacific Ocean, DMS concentrations ranging from 0.2 to $400 \times 10^{-9} \mathrm{~g} \mathrm{~m}^{-3}$, from Panama to Hobart (B. C. Nguyen et al., unpublished manuscript, 1983).

2. $\mathrm{SO}_{2}$ formation above the ocean: (a) Mechanisms. Organic sulfides released from the ocean surface are rapidly oxidized in the marine atmosphere. Bentley et al. [1972], Cox [1975], Slagle et al. [1976], Kurylo [1978b], and Grosjean and Lewis [1982] proposed several likely oxidation mechanisms by $\mathrm{O}^{\cdot}$ and $\mathrm{OH}^{\cdot}$ photochemically produced in the atmosphere. The following reactions summarize how $\mathrm{SO}_{2}$ may be produced from DMS and methylmercaptan:

$$
\begin{gathered}
\mathrm{CH}_{3} \mathrm{SCH}_{3}+\mathrm{O} \cdot \mathrm{CH}_{3} \mathrm{O}+\mathrm{CH}_{3} \mathrm{~S} \\
k=6.3 \pm 1.3 \times 10^{-11} \mathrm{~cm}^{3} \text { molecule }{ }^{-1} \mathrm{~s}^{-1} \\
\mathrm{CH}_{3} \mathrm{SCH}_{3}+\mathrm{HO} \rightarrow \text { products } \\
k=1.5 \times 10^{-11} \mathrm{~cm}^{3} \text { molecule }{ }^{-1} \mathrm{~s}^{-1} \\
\mathrm{CH}_{3} \mathrm{SH}+\mathrm{HO} \rightarrow \mathrm{H}_{2} \mathrm{O}+\mathrm{CH}_{3} \mathrm{~S} \\
k=1.4 \times 10^{-10} \mathrm{~cm}^{3} \text { molecule }{ }^{-1} \mathrm{~s}^{-1} \\
\mathrm{CH}_{3} \mathrm{~S}+\mathrm{O}_{2} \rightarrow \mathrm{CH}_{3} \mathrm{SO}_{2} \rightarrow \mathrm{CH}_{3}+\mathrm{SO}_{2} \\
\mathrm{CH}_{3} \mathrm{SO}_{2} \rightarrow \mathrm{CH}_{3} \mathrm{SO} \rightarrow \mathrm{CH}_{3} \mathrm{O}+\mathrm{SO}_{2}
\end{gathered}
$$

$$
\begin{aligned}
\mathrm{CH}_{3} \mathrm{SO} & \rightarrow \mathrm{SO}+\underset{\mathrm{CH}_{3}}{\stackrel{\mathrm{O}_{2}}{\rightarrow}} \mathrm{SO}_{2}+\mathrm{CH}_{3} \mathrm{O} \cdot \\
& \stackrel{\mathrm{O}_{2}}{\rightarrow} \mathrm{SO}_{3} \stackrel{\mathrm{NO}}{\rightarrow} \mathrm{SO}_{2}
\end{aligned}
$$

Furthermore, other pathways lead to different gaseous or particulate sulfur compounds qualitatively identified by Bentley et al. [1972], Hatakeyama et al. [1982], and Grosjean and Lewis [1982], such as:

$$
\begin{gathered}
\mathrm{CH}_{3} \mathrm{SOCH}_{3}, \mathrm{CH}_{3} \mathrm{SO}_{3} \mathrm{H}, \mathrm{CH}_{3} \mathrm{SOH}, \mathrm{H}_{2} \mathrm{SO}_{4}, \mathrm{SO}_{3} \\
\mathrm{CH}_{3} \mathrm{SO}_{2} \mathrm{CH}_{3}, \mathrm{CH}_{3} \mathrm{SSCH}_{3},\left(\mathrm{CH}_{3} \mathrm{O}\right)_{2} \mathrm{SO}_{2}, \mathrm{CH}_{3} \mathrm{OSO}_{2} \mathrm{CH}_{3}
\end{gathered}
$$

From Hatakeyama et al. [1982], only $21 \%$ of the DMS is oxidized into $\mathrm{SO}_{2}$, whereas greater yields are obtained by Bentley et al [1972], who found $31 \%$, and by Grosjean and Lewis [1982], who found 35 to $73 \%$. Thus, $\mathrm{SO}_{2}$ is always a product of the photooxidation of DMS but with a yield closely depending on the laboratory conditions, particularly in the initial contents in DMS (several orders of magnitude greater than the natural atmospheric contents).

(b) DMS and $\mathrm{SO}_{2}$ concentrations over a high-productivity area; evidence of an "in situ" photooxidation of DMS. Two different experiments were performed in a coastal site of Guisseny (Brittany, France), which is rich in algae (Polysiphonia lanosa and laminaria), known to produce important amounts of DMS (Figure 2).

During the first experiment, previously described by Bonsang et al. [1976a], $\mathrm{SO}_{2}$ was sampled at site $\mathrm{B}, 200$ meters inland in the direction of the dominant wind flowing above the algae beds (Figure 2).

We observed a diurnal effect in the $\mathrm{SO}_{2}$ production; the atmospheric $\mathrm{SO}_{2}$ concentration during the day reached values as high as $6 \mu \mathrm{g} / \mathrm{m}^{3}$, while concentrations at night varied between 0.2 and $1.8 \mu \mathrm{g} / \mathrm{m}^{3}$ [Bonsang et al., 1976a], (Figure 3). Diurnal maxima were observed at the low-tide hours and increased with the tidal coefficient.

Furthermore, the $\mathrm{SO}_{2}$ concentrations were sharply correlated with the Aitken nucleii production, which reached values close to $10^{6} / \mathrm{cm}^{3}$ at low-tide hours [Paugam et al., 1977].

The atmospheric $\mathrm{SO}_{2}$ concentration seems to increase with both the uncovered algae areas and the sunlight intensity, these experiments in coastal areas near important sources of dimethylsulfide argue persuasively that the $\mathrm{SO}_{2}$ production is photochemically mediated.

In a second experiment, in February 1978, we simultaneously measured DMS on site B previously described and on site $A$ at about $30 \mathrm{~cm}$ above the algae beds shown in Figure 2 . The atmospheric concentration of DMS at sites A and B in February 1978 were $80 \times 10^{-9} \mathrm{~g} / \mathrm{m}^{3}$ and $2.7 \times 10^{-9} \mathrm{~g} / \mathrm{m}^{3}$, respectively. Such a decrease in DMS concentration between sites A and $B$ (factor of 30) confirms a local DMS production.

It may be suggested that the diurnal $\mathrm{SO}_{2}$ effect previously observed is very likely caused by this biogenic local production of DMS or of other sulfide compounds not yet investigated. However, to explain $\mathrm{SO}_{2}$ concentrations as high as 6 $\mu \mathrm{g} / \mathrm{m}^{3}$, we have to admit very fast oxidation rates for these particular conditions, since the transport time between the source of sulfide compounds (site $\mathrm{A}$ ) and the $\mathrm{SO}_{2}$ measurement (site B) do not exceed $2 \mathrm{~min}$ (wind speed $<5 \mathrm{~m} \mathrm{~s}^{-1}$ ).

The occurrence of high concentrations of DMS in open oceanic areas, as well as these last experiments in a coastal site, suggest that DMS could be the predominant precursor for marine $\mathrm{SO}_{2}$. This hypothesis is apparently only consistent 


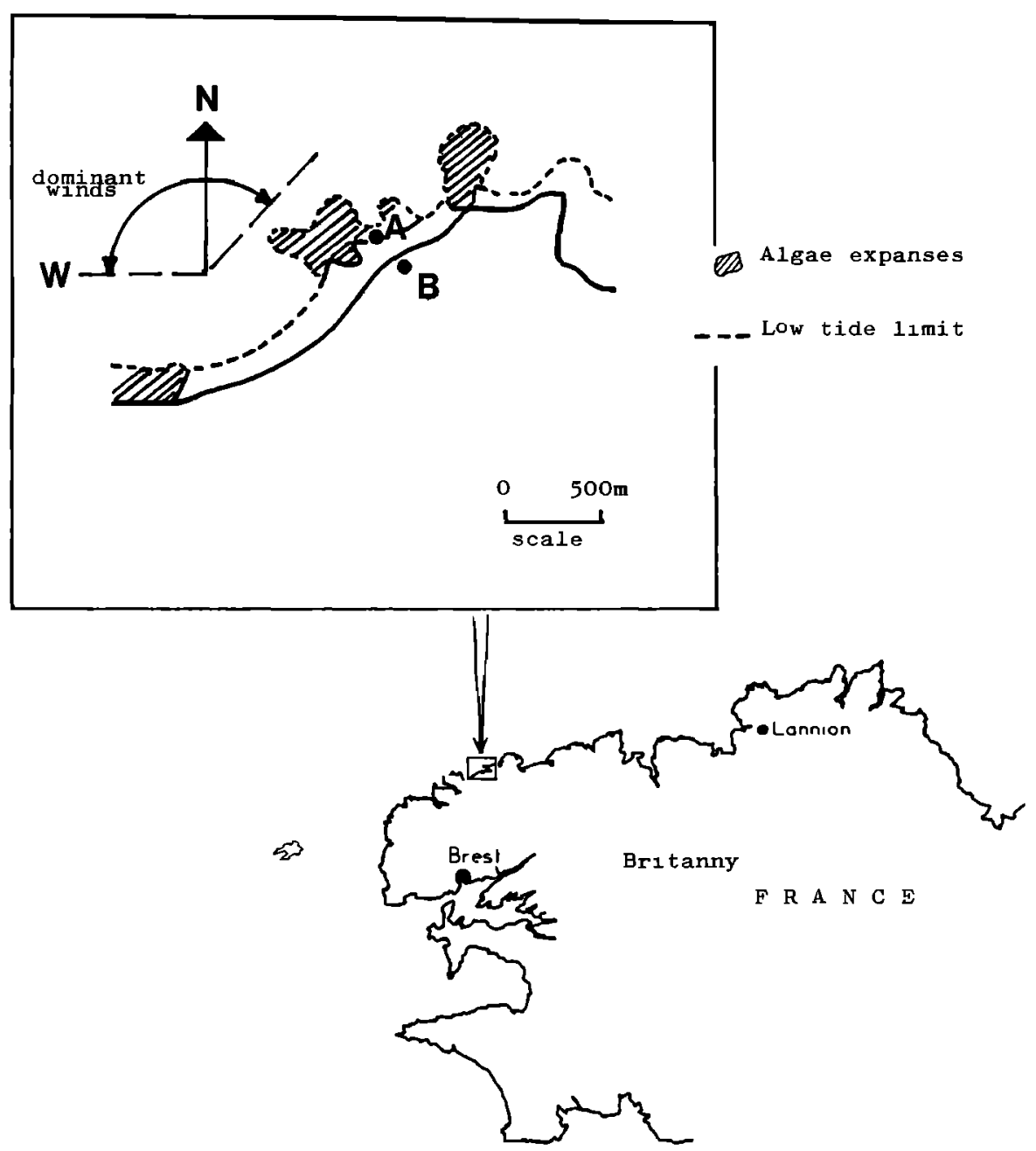

Fig. 2. Site of Guisseny (Brittany, France).

with a rate of oxidation of DMS that is higher than current laboratory measurements suggest. However, other organic sulfur compounds, more reactive than DMS, cannot be discarded as potential precursors of $\mathrm{SO}_{2}$. Field data as presented could now corroborate both these hypotheses.

The Influence of the Biological Activity on the ProDUCtion of Sulfur DioXIDE IN THE MARINe ATMOSPHERE

1. Atmospheric concentration of $\mathrm{SO}_{2}$ in upwelling and convergence areas. It is well known that upwelling areas have high levels of biological activity. Two cruises, A and G (Figure 1a), were made in the Gulf of Guinea upwelling area. $\mathrm{SO}_{2}$ concentrations observed in March 1973 and December 1976 between Dakar and Capetown were always higher than the mean $0.1 \mu \mathrm{g} \mathrm{SO}_{2} / \mathrm{m}^{3}$ ocean value and ranged from 0.2 to 0.45 $\mu \mathrm{g} / \mathrm{m}^{3}$ inside the upwelling area; lower values, from 0.04 to $0.10 \mu \mathrm{g} / \mathrm{m}^{3}$, were found outside the main upwelling area.

A third cruise in the Peruvian upwelling (Figure 1a) confirmed these results: $\mathrm{SO}_{2}$ concentrations ranged from 0.2 to $0.7 \mu \mathrm{g} / \mathrm{m}^{3}$ in the upwelling area and from 0.1 to $0.15 \mu \mathrm{g} / \mathrm{m}^{3}$ outside [Bonsang et al., 1976b; Bonsang, 1980]. In addition, the influence of biological activity on atmospheric $\mathrm{SO}_{2}$ con-

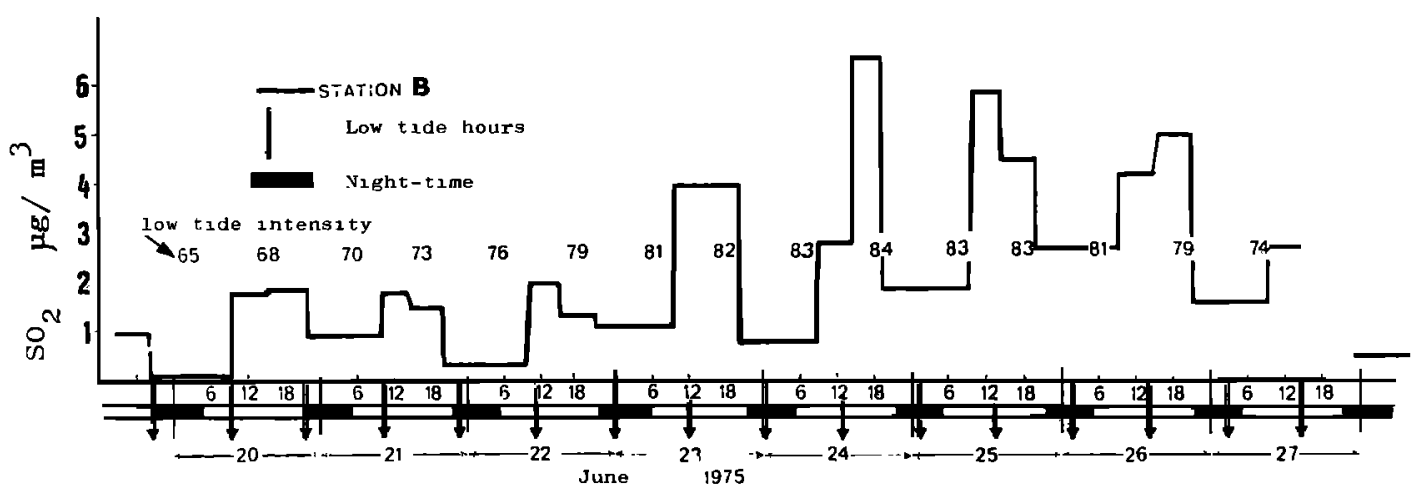

Fig. 3. Atmospheric $\mathrm{SO}_{2}$ concentrations at Guisseny (site B), June 1975. 

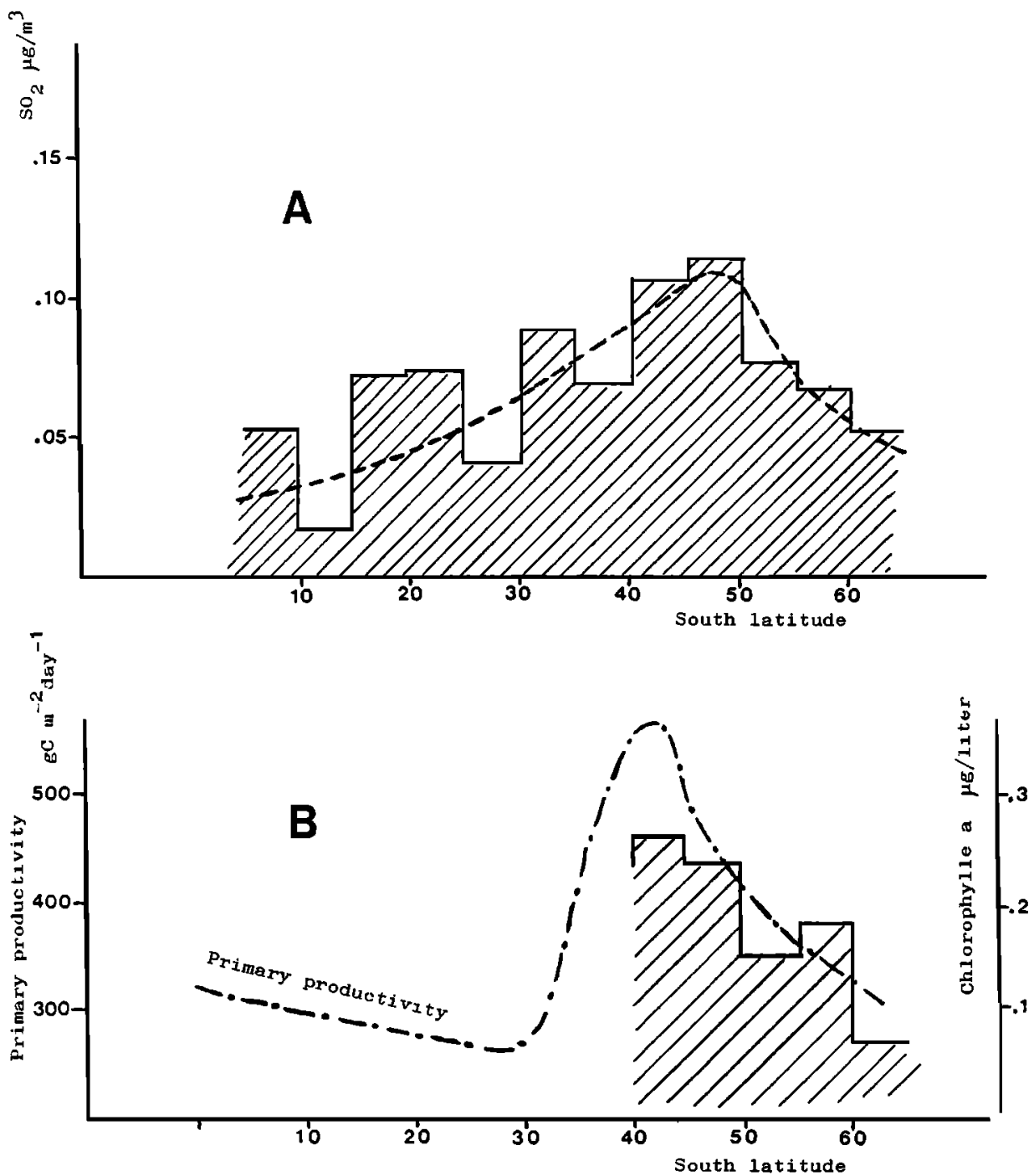

Fig. 4. (a) Atmospheric $\mathrm{SO}_{2}$ concentration $\left(\mu \mathrm{g} / \mathrm{m}^{3}\right)$ versus latitude in the southern part of the Indian Ocean between $55^{\circ} \mathrm{E}$ and $75^{\circ} \mathrm{E}$. (b) Simultaneous record of chlorophyll "a" (solid line). Also shown is primary productivity observed above the $70^{\circ} \mathrm{E}$ meridian at the same time of year (late summer), from Krey [1973] (dashed line).

centrations has been studied in convergence areas, where primary productivity is higher than in tropical regions. This influence is illustrated in Figure 4, which shows the mean atmospheric $\mathrm{SO}_{2}$ concentration in each $5^{\circ}$ of latitude between $5^{\circ} \mathrm{S}$ and $62^{\circ} \mathrm{S}$. This figure was obtained from 138 measurements in the Indian Ocean performed during cruises of the R/V Marion Dufresne from 1973 to 1977 . We can observe that the $\mathrm{SO}_{2}$ concentration reaches a maximum of $0.12 \mu \mathrm{g} / \mathrm{m}^{3}$ near the Antarctic convergence zone $\left(45-50^{\circ} S\right)$ and decreases to $0.05 \mu \mathrm{g} / \mathrm{m}^{3}$ both southward and northward.

This latitudinal dependence of atmospheric $\mathrm{SO}_{2}$ concentrations is thus very well related to the general variation of primary productivity. Particularly between $40^{\circ} \mathrm{S}$ and $60^{\circ} \mathrm{S}$, we can observe the same trend of variation between atmospheric $\mathrm{SO}_{2}$ concentrations and the chlorophyll "a" concentrations simultaneously measured in sea surface water. (Collaboration with the Laboratoire Arago at Banyuls-sur-Mer, G. Jacques and $\mathrm{G}$. Neveu). The large variation of $\mathrm{SO}_{2}$ measurements is also quite reasonable, since the concentrations should be greatly influenced by chemical factors producing and destroying $\mathrm{SO}_{2}$ and, perhaps, meteorological factors diluting the substance.

2. Atmospheric $\mathrm{SO}_{2}$ concentrations versus primary productiv- ity in several oceanic areas. From $162 \mathrm{SO}_{2}$ measurements we can represent the atmospheric $\mathrm{SO}_{2}$ concentration above different oceanic areas versus primary productivity in the sea surface water (Figure 5). We observe that the weak productivity areas, such as the tropical Indian Ocean (primary production less than $36 \mathrm{~g} \mathrm{~cm}^{-2} \mathrm{yr}^{-1}$ correspond to a low $\mathrm{SO}_{2}$ concentration of $0.03 \mu \mathrm{g} / \mathrm{m}^{3}$; areas with an intermediate primary productivity between 36 and $90 \mathrm{~g} \mathrm{~cm}^{-2} \mathrm{yr}^{-1}$ have an atmospheric $\mathrm{SO}_{2}$ concentration of about $0.1 \mu \mathrm{g} / \mathrm{m}^{3}$; regions of highest production (convergence and upwelling areas greater than $90 \mathrm{~g} \mathrm{~cm}^{-2} \mathrm{yr}^{-1}$ ) correspond to $0.2-0.3 \mu \mathrm{g} \mathrm{SO}_{2} / \mathrm{m}^{3}$.

\section{Flux of Sulfur Compounds from the OcEAN to THE ATMOSPHERE}

At high altitude the $\mathrm{SO}_{2}$ concentrations observed in the marine atmosphere can be considered as resulting from a steady state balance between the oxidation of reduced sulfur compounds and the oxidation of $\mathrm{SO}_{2}$ to sulfate by gas-toparticle conversion. The flux of sulfur compounds from the ocean to the atmosphere can be calculated from the atmospheric $\mathrm{SO}_{2}$ profile and its residence time above the ocean. Numerous $\mathrm{SO}_{2}$ profiles have been established above the polluted mid-latitude European continents and show an ex- 


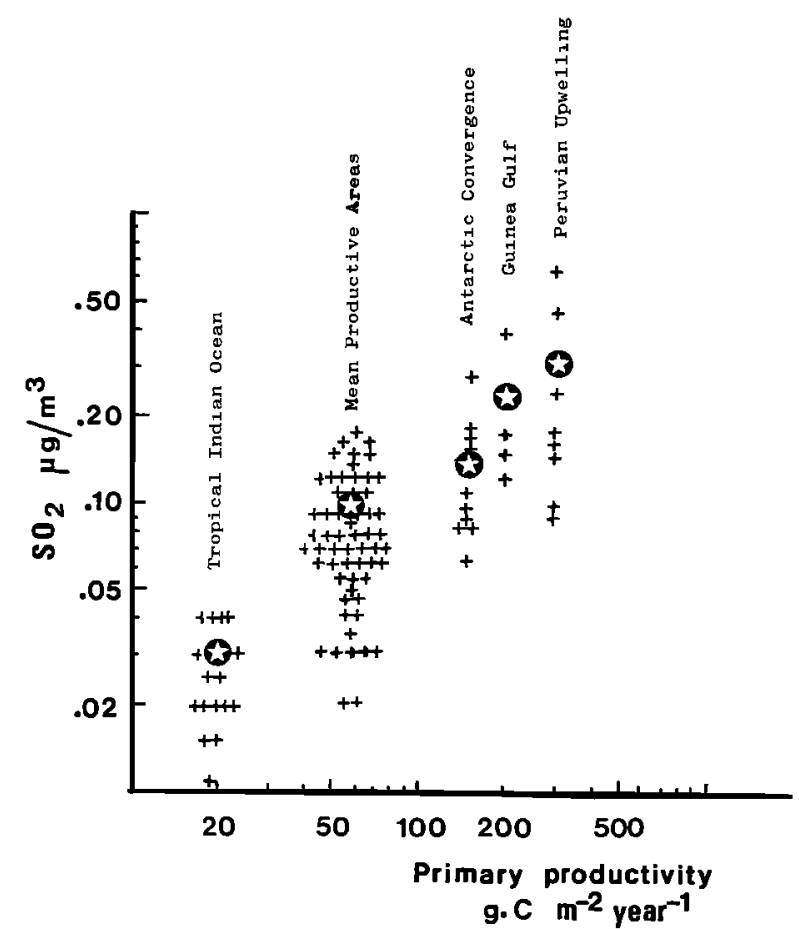

Fig. 5. $\mathrm{SO}_{2}$ concentration variations with respect to primary productivity in several oceanic regions.

ponential decrease versus the altitude [Rodhe 1972; Jost, 1974; Jaeschke et al., 1976; Varhelyi, 1978], whereas $\mathrm{SO}_{2}$ profiles above the oceans are very sparse.

However, such a profile has been established by Georgii and Meixner [1980] above the Atlantic, close to the west of France. This profile shows a practically constant mixing ratio of $\mathrm{SO}_{2}\left(100 \mathrm{pptv}\right.$ or $0.27 \mu \mathrm{g} \mathrm{SO}_{2} / \mathrm{STD} \mathrm{m}{ }^{3}$ ) from 1000 to 6500 $\mathrm{m}$. Such values were later confirmed by Maroulis and Bandy [1980], who indicated in the free troposphere a mean concentration level of $122 \mathrm{pptv}$ in the northern hemisphere and 90 pptv in the southern hemisphere.

In the lower marine atmosphere, scavenging processes of $\mathrm{SO}_{2}$ are much more efficient than in the free troposphere because the ocean surface acts as a sink for this gas [Beilke and Lamb, 1974]. Consequently, the $\mathrm{SO}_{2}$ concentration may decrease toward the ocean atmosphere interface. Both the average $\mathrm{SO}_{2}$ concentration of $35 \mathrm{pptv}$, measured at the sea level by $N$ guyen et al. $[1974 a, b]$, and the slightly higher mean concentration of 57 pptv in the boundary layer, reported by Maroulis et al. [1980], tend to confirm that effect.

Hence, we can consider two different cases:

1. For an oceanic source of $\mathrm{SO}_{2}$, caused by the oxidation of reduced sulfur compounds outgased from the sea surface, the atmospheric concentration of $\mathrm{SO}_{2}$ would increase up to altitude $h_{1}$ and then decrease exponentially (Figure 6, curve A), according to the profile proposed by Logan et al. [1979]. Indeed, our previous study [Nguyen, 1968; Nguyen and Lambert, 1970] concerning the exchanges between the stratosphere and the troposphere suggests that similar mixing conditions occur for gases and particles beyond the boundary layer of both oceanic and continental areas; therefore, $\mathrm{SO}_{2}$ concentrations should decrease exponentially over the oceans as well as over continents.

2. For meteorological situations typical of the tropics and, possibly, portions of the midlatitudes, $\mathrm{SO}_{2}$ could be produced at low altitude and transported, as well as its precursors, throughout the troposphere [Chatfield and Crutzen, 1981]. Their vertical profiles depend on the presence of cumulonimbus clouds scattered through a region occupying about $20 \%$ of a larger synoptic region, more or less as is observed. Hence the vertical mixing ratio of $\mathrm{SO}_{2}$ can increase up to 1000 or 2000 meters of altitude and stay almost constant up to 10,000 meters (curve B).

Since the experimental values are located between these two extreme cases, we will consider them in developing an estimate of the $\mathrm{SO}_{2}$ budget.

Case 1: Increase of $\mathrm{SO}_{2}$ concentration from $0.1 \mu \mathrm{g} / \mathrm{m}^{3}$ STD at sea level to $0.16 \mu / \mathrm{m}^{3}$ STD at altitude $h_{1}$ (close to $1500 \mathrm{~m}$ ), then exponential decrease.

Case 2: Same variations versus altitude in the low troposphere up to altitude $h_{1}$, then the mixing ratio remains constant from altitude $h_{1}$ to $10 \mathrm{~km}$.

Hence from sea level to altitude $h_{1}$ the $\mathrm{SO}_{2}$ mixing ratio follows the general law:

$$
\left(\mathrm{SO}_{2}\right)_{h}=\left(\mathrm{SO}_{2}\right)_{0} e^{\beta h}
$$

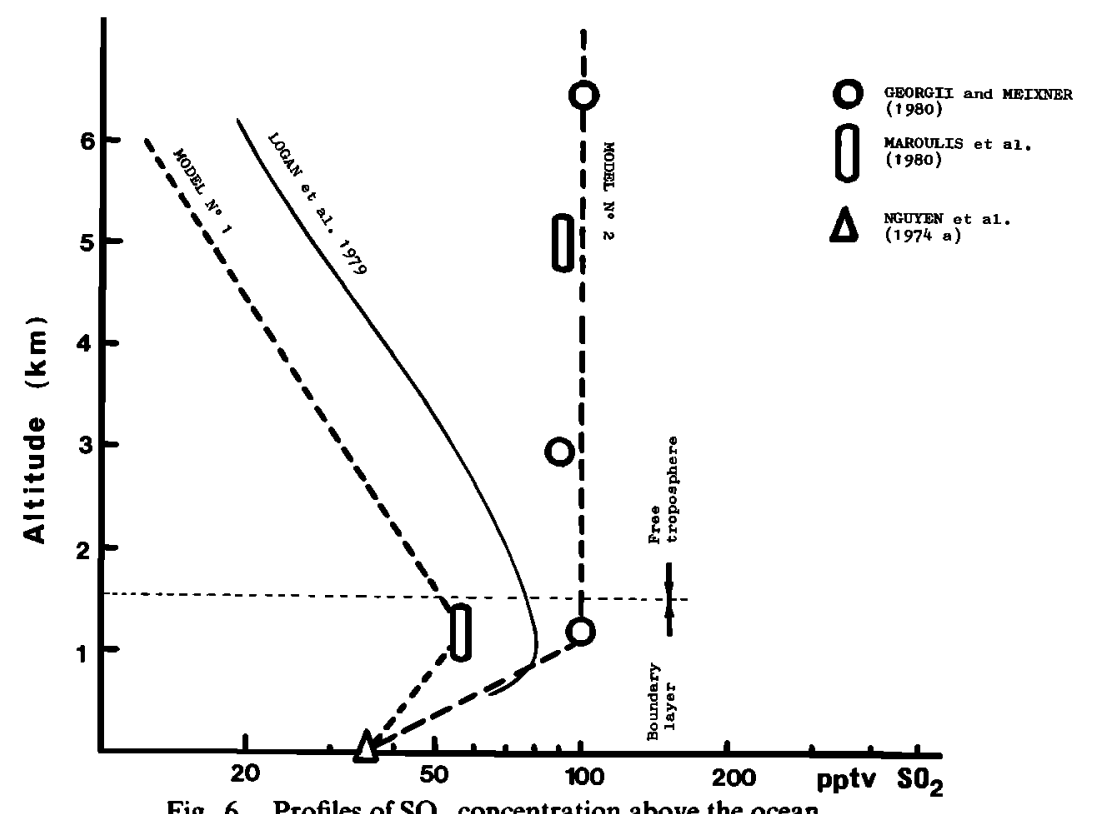

Fig. 6. Profiles of $\mathrm{SO}_{2}$ concentration above the ocean. 
Beyond altitude $h_{1}$, the $\mathrm{SO}_{2}$ mixing ratio varies according to

$$
\left(\mathrm{SO}_{2}\right)_{h}=\left(\mathrm{SO}_{2}\right) h_{1} e^{-a\left(h-h_{1}\right)}
$$

( $\alpha=0$ in model 2 ).

The total quantity $Q$ of $\mathrm{SO}_{2}$ in an air column of unit area above the ocean is then equal to

$$
Q=Q_{1}+Q_{2}
$$

with

$$
\begin{gathered}
Q_{1}=\int_{0}^{h_{1}} C_{0} e^{\beta h} \frac{\rho(h)}{\rho_{0}} S d h \\
Q_{2}=\int_{h_{1}}^{h_{2}} C_{h_{1}} e^{-a\left(h-h_{1}\right)} \frac{\rho(h)}{\rho_{0}} S d h
\end{gathered}
$$

where $\rho(h)$ is the air density at altitude $h$, i.e., $\rho(h)=\rho_{0} e^{-h / H}$, where $\rho_{0}$ is the air density at level 0 , and where $H$, the scale height constant is $7936 \mathrm{~m} . S$ is the total surface of the oceans, $360 \times 10^{12} \mathrm{~m}^{2} ; \mathrm{C}_{0}=0.1 \mu \mathrm{g} / \mathrm{m}^{3}$ (this study) $\beta$ is calculated as $\beta=1 / h_{1} \operatorname{Ln}\left(C_{h_{1}} / C_{0}\right) ; C_{h_{1}}=0.16 \mu \mathrm{g} / \mathrm{m}^{3}$ [Maroulis et al., 1978], (model 1); $C_{h_{2}}=0.27 \mu \mathrm{g} / \mathrm{m}^{3}$ [Georgii and Meixner, 1980], (model 2); and $h_{1}$ is taken as $1500 \mathrm{~m}$.

An average $\alpha=3.95 \times 10^{-4} / \mathrm{m}$ is calculated from nine profiles of $\mathrm{SO}_{2}$ concentration observed by Georgii $[1970,1978]$, Rodhe [1972], and Jost [1974] by the relation

$$
\alpha=\frac{1}{h} \operatorname{Ln} \frac{C_{h}}{C_{\text {ground }}}
$$

The flux of gaseous sulfur compounds from the ocean to the atmosphere is then

$$
F=\frac{Q_{1}}{\tau_{1}}+\frac{Q_{2}}{\tau_{2}}
$$

where $\tau_{1}$ and $\tau_{2}$ are the respective residence times of $\mathrm{SO}_{2}$ in the boundary layer and the free troposphere. $Q_{1}$ and $Q_{2}$ are equal to 3.1 and $4.6 \times 10^{10} \mathrm{~g} \mathrm{~S}$ in model 1 and 4.1 and $21.0 \times$ $10^{10} \mathrm{~g} \mathrm{~S}$ in model 2 , respectively.

The flux of $\mathrm{SO}_{2}(Q / \tau)$ produced from the ocean surface depends on the residence time $\tau$ of this gas in a given reservoir. In the low atmosphere we have determined a value $\tau_{1}$ close to 0.7 day [Nguyen et al., 1975], resulting from both sulfate oxidation and direct absorption by seawater. In the upper troposphere, above the boundary layer, only the oxidation of $\mathrm{SO}_{2}$ into sulfate is the dominant process. Unfortunately, no experimental data for $\mathrm{SO}_{2}$ residence time is available in the free troposphere.

The data of Prahm et al. [1976] and Smith and Jeffrey [1975] indicate an oxidation rate of $\mathrm{SO}_{2}$ comprising between $1.1 \times 10^{-2} \mathrm{~h}^{-1}$ and $2.1 \times 10^{-2} \mathrm{~h}^{-1}$. Our previous works suggest an oxidation rate close to $4 \times 10^{-2} \mathrm{~h}^{-1}$ deduced from $\mathrm{SO}_{4} / \mathrm{SO}_{2}$ ratio in the marine atmosphere [Bonsang et al., 1980]. Hence we can admit an average value of the order of $2.3 \times 10^{-2} \mathrm{~h}^{-1}$ for the oxidation rate of $\mathrm{SO}_{2}$ into sulfate, which leads to a residence time close to 1.5 days in the free troposphere.

This last value seems reasonable for the free troposphere between 2- and 6-km altitudes, and it has been also used by Rodhe and Isaksen [1980]. This can be argued by the fact that heterogeneous oxidation processes occur in the cloudy layer as well as at the marine boundary.

Thus models 1 and 2 lead to fluxes of 27 and $72 \times 10^{6}$ tons of sulfur per year, respectively, outgased from the ocean to the atmosphere. The only experimental profile in marine atmosphere is better described by model 2 . If such a profile was confirmed, the flux of biogenic marine sulfur would be close to the highest value of our estimate.

\section{Conclusions}

Many questions still remain about the sulfur cycle in marine areas, which cover three quarters of the earth's surface. In particular, sulfur compound altitude profiles above remote oceanic areas could add a lot to the knowledge about the sulfur cycle. In spite of these problems we believe that it is possible to draw from this study the following conclusions:

1. A background concentration of $0.1 \mu \mathrm{g} / \mathrm{m}^{3}$ (35 pptv) of $\mathrm{SO}_{2}$ has been confirmed above different oceanic areas far from any continental influences.

2. Several possible origins for $\mathrm{SO}_{2}$ have been advanced. Instead, we have shown here that this background comes principally from oceanic biological activity, which produces volatile sulfur compounds, mainly $\mathrm{CH}_{3} \mathrm{SCH}_{3}$ and probably also $\mathrm{CH}_{3} \mathrm{SH}$ and $\mathrm{CH}_{3} \mathrm{SSCH}_{3}$, outgased at the ocean surface. In turn these are partly oxidized in the atmosphere to $\mathrm{SO}_{2}$ and sulfate by various photochemically mediated mechanisms, as outlined in Figure 7.

Among these organic sulfides, dimethylsulfide (DMS) has been made conspicuous in the ocean water at all depths (10 to several hundreds of $\left.10^{-9} \mathrm{~g} / \mathrm{l}\right)$ as well as in the atmosphere above the water $\left(0.2\right.$ to $\left.400 \times 10^{-9} \mathrm{~g} / \mathrm{m}^{3}\right)$. Other sulfur compounds, such as $\mathrm{COS}$ and $\mathrm{CS}_{2}$, occuring in the marine atmosphere could not explain more than $20 \%$ of the measured $\mathrm{SO}_{2}$. Concerning $\mathrm{H}_{2} \mathrm{~S}$, no data exist in the very remote subantarctic areas, and it cannot be excluded that this compound remains a possible precursor of this marine $\mathrm{SO}_{2}$.

3. The emission of sulfur compounds produced by biological mechanisms has been estimated from 27 to $72 \times 10^{6}$ tons of sulfur per year. This estimate, based on the vertical profile of atmospheric $\mathrm{SO}_{2}$, may be considered as an approximation because of the lack of data in marine atmospheres. More data is needed to support our results.

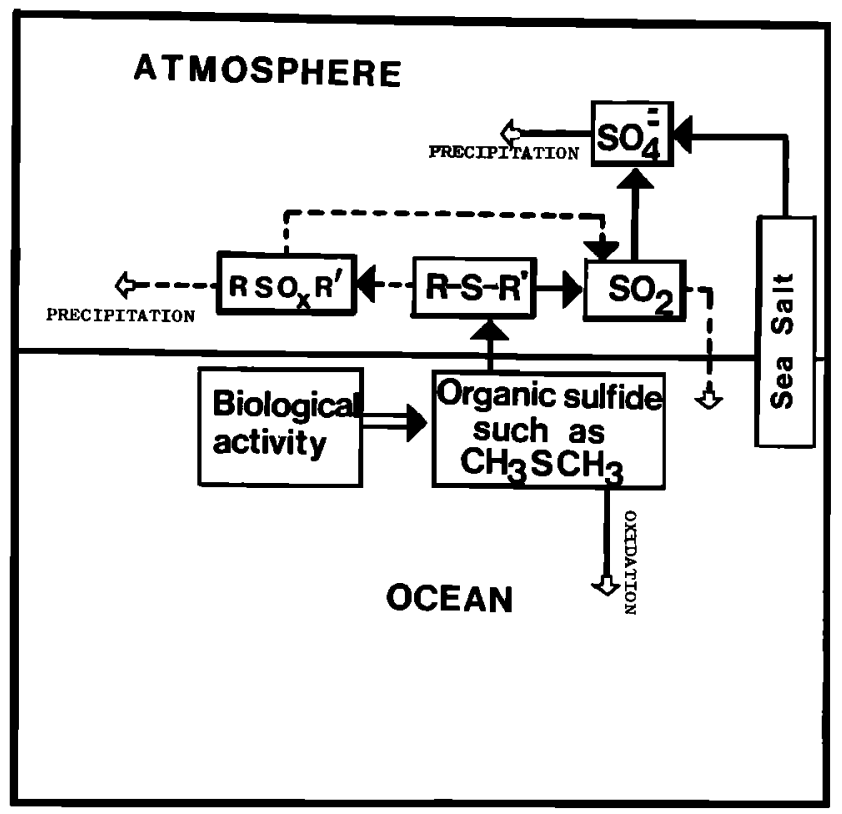

Fig. 7. Scheme for sulfur compound exchange between the ocean and the atmosphere leading to sulfate production by gas-to-particle conversion. Units are $10^{6}$ tons of sulfur/year. 
According to Woodwell et al. [1978], the primary production for the whole ocean is $25 \times 10^{15} \mathrm{~g}$ of carbon per year, which corresponds to a mean productivity of $70 \mathrm{~g} \mathrm{~cm}^{-2} \mathrm{yr}^{-1}$. By referring to Figure 5, this value does correspond to the mean $\mathrm{SO}_{2}$ concentration of $35 \mathrm{pptv}$, which we found and which appears reasonable in our flux calculations.

This flux is of the same order as the sea spray production of $44 \times 10^{6}$ tons of sulfur per year [Eriksson, 1960] and anthropogenic inputs (50-80 × $\left.10^{6} \mathrm{~T} \mathrm{~S} / \mathrm{yr}\right) ;$ [Robinson and Robbins, 1970; Kellogg et al., 1972; Friend, 1973; Granat et al., 1976; Rodhe and Isaksen, 1980], but is much greater than the flux from volcanos (2-3 $\left.\times 10^{6} \mathrm{~T} \mathrm{~S} / \mathrm{yr}\right)$ [Stoiber and Jepsen, 1973; Granat et al., 1976]. Therefore, the contribution of the ocean could be estimated within 71 and $116 \times 10^{6} \mathrm{~T} \mathrm{~S} / \mathrm{yr}$.

4. Concerning the oceanic emission of sulfur compounds, the sea spray production leads to the formation of aerosols of relatively great size which quickly fall back into the sea or on the continent near the coast. Besides, biological sources lead to the formation of submicronic aerosols by gas-to-particle conversion [Bonsang et al., 1980].

The simultaneous increase of $\mathrm{SO}_{2}$ and Aitken nucleii concentrations, which we observed at low tide above an algae bed contribute to reinforce that hypothesis.

These particles could have long enough residence times in the atmosphere to feed the high troposphere and, perhaps, the stratospheric reservoir. Moreover, because of their hygroscopicity, sulfate aerosols produced by this process could play an important role as condensation nucleii in the formation of clouds. Biological activity could thus have an indirect influence on climate.

Acknowledgments. We are grateful to $\mathrm{G}$. Lambert for suggestions and helpful discussions, R. Chatfield and J. D. Shetter for interesting discussions and reviewing the manuscript, and to $C$. Lorius and his team for sampling in Antarctica. We also thank Ministère de l'Environnement et du Cadre de Vie, Centre National pour l' Exploitation des Océans, Centre National de la Recherche Scientifique, Terres Australes et Antarctiques Francaises, and Expéditions Polaires Francaises, which supported this study.

\section{REFERENCES}

Andreae, M. O., Dimethylsulfoxide in marine and freshwater, Limnol. Oceanogr., 25, 1054-1063, 1980.

Armstrong, F. A. J., and G. T. Boalch, Volatile organic matter in algae culture media and sea water, Nature, 185, 761-762, 1960.

Barnard, W. R., M. O. Andreae, W. E. Watkins, H. Bingermer, H. W. Georgii, The flux of dimethylsulfide from the oceans to the atmosphere, J. Geophys. Res., 87, 8787-8793, 1982.

Beilke, S., and D. Lamb, On the absorption of $\mathrm{SO}_{2}$ in ocean water, Tellus, 26, 268-271, 1974.

Bentley, M. D., I. B. Douglass, J. A. Lacadie, and D. R. Whittier, The photolysis of dimethyl sulfide in air, J. Air Pollut. Contr. Assoc., 22, 359-363, 1972.

Bonsang, B., Cycle atmosphérique due soufre d'origine marine, $\mathrm{Ph} . \mathrm{D}$. thesis, Univ. Picardie, St. Quentin, France, 1980.

Bonsang, B., and B. C. Nguyen, Sulfur dioxide measurements in marine atmosphere at concentrations lower than ppb, Proceedings of 13th International Colloquim, Paris, in Atmospheric Pollution 1978 , edited by M. M. Benarie, pp. 77-79, Elsevier, New York, 1978.

Bonsang, B., B. C. Nguyen, and J. Y. Paugam, Sur la formation de gaz sulfurés et de noyaux d'Aitken dans l'atmosphère le long du littoral, C. R. Acad. Sci. Paris, Ser. D, 283, 1285-1288, 1976a.

Bonsang, B., B. C. Nguyen, and G. Lambert, Biological activity influence on atmospheric $\mathrm{SO}_{2}$ of marine origin, paper presented at Joint Oceanographic Assembly, Edinburgh, $1976 b$.

Bonsang, B., B. C. Nguyen, A. Gaudry, and G. Lambert, Sulfate enrichment in marine aerosols due to biogenic gaseous sulfur compounds, J. Geophys. Res., 85, 7410-7416, 1980.
Chameides, W. L., and A. Tan, The two-dimensional diagnostic model for tropospheric OH: An uncertainty analysis, J. Geophys. Res., 86, 5209-5223, 1981.

Chatfield, R. B., and P. J. Crutzen, Marine sulfur emissions, $\mathrm{SO}_{2}$ in the clean upper troposphere, and the global sulfur budget, paper presented at 3rd Scientific Assembly, IAMAP, Hamburg, Fed. Repub. Ger., 1981.

Cox, R. A., Atmospheric photo-oxidation reactions: The gas phase reaction of $\mathrm{OH}$ radicals with some sulfur compounds, U.K. At Energy Res. Estab., Rep. 8132, 1-10, 1975.

Crutzen, P. J., The possible importance of OCS for the sulfate layer of the stratosphere, Geophys. Res. Lett., 3, 73-76, 1976.

Delmas, R., and J. Servant, The origins of sulfur compounds in the atmosphere of a zone of high productivity (Gulf of Guinea), $J$. Geophys. Res., 87, 11,019-11,026, 1982.

Eriksson, E., The yearly circulation of chloride and sulfur in nature; Meteorological, geochemical, and pedological implications: Part 2 , Tellus, 12, 63-109, 1960.

Eriksson, E., The yearly circulation of sulfur in nature, J. Geophys. Res., 68, 4001-4008, 1963.

Friend, J. P., The global sulfur cycle, in Chemistry of the Lower Atmosphere, edited by S. I. Rasool, pp. 177-201, New York, 1973.

Georgii, H. W., Contribution to the atmospheric sulfur budget, $J$. Geophys. Res., 75, 2365-2371, 1970.

Georgii, H. W., Spatial and temporal distribution of sulfur compounds, Intervational Symposium on Sulfur in the Atmosphere, Dubrovnik (Yugoslavia), Atmos. Environ., 12, 681-690, 1978.

Georgii, H. W., and F. X. Meixner, Measurement of the tropospheric and stratospheric $\mathrm{SO}_{2}$ distribution, J. Geophys. Res., 85, 74337438, 1980.

Granat, L., H. Rodhe, and R. O. Hallberg, The global sulfur cycle, Ecol. Bull., 22, 89-134, 1976.

Grosjean, D., and R. Lewis, Atmospheric photooxidation of methyl sulfide, Geophys. Res. Lett., 9, 1203-1206, 1982.

Hatakeyama, S., M. Okuda, and H. Akimoto, Formation of sulfur dioxide and methane sulfonic acid in the photooxidation of dimethyl sulfide in the air, Geophys. Res. Lett., 9, 583-586, 1982.

Jaeschke, W., R. Schmitt, and H. W. Georgii, Preliminary results of stratospheric $\mathrm{SO}_{2}$ measurements, Geophys. Res. Lett., 3, 517-519, 1976.

Jost, D., Aerological studies on the atmospheric sulfur budget, Tellus, 26, 206-212, 1974

Kellogg, W. W., R. D. Cadle, E. R. Allen, A. L. Lazrus, and E. A. Martell, The sulfur cycle, Science, 175, 587-596, 1972

Kurylo, M. J., Flash photolysis resonance fluorescence investigation of the reaction of $\mathrm{OH}$ radicals with OCS and $\mathrm{CS}_{2}$, Chem. Phys. Lett., 58, 238-242, 1978a.

Kurylo, M. J., Flash photolysis resonance fluorescence investigation of the reaction of $\mathrm{OH}$ radicals with dimethyl sulfide, Chem. Phys. Lett., 58, 233-237, 1978b.

Logan, J. A., M. B. McElroy, S. C. Wofsy, and M. J. Prather, Oxidation of $\mathrm{CS}_{2}$ and $\mathrm{COS}$ : Sources for atmospheric $\mathrm{SO}_{2}$, Nature, 281, 185-188, 1979.

Lovelock, J. E., R. J. Maggs, and R. A. Rasmussen, Atmospheric dimethyl sulfide and the natural sulfur cycle, Nature, 237, 452-453, 1972.

Maroulis, P. J., and A. R. Bandy, Estimate of the contribution of biologically produced dimethyl sulfide to the global sulfur cycle, Science, 196, 647-648, 1977.

Maroulis, P. J., A. L. Torres, A. B. Goldberg, and A. R. Bandy, Measurements of tropospheric background levels of $\mathrm{SO}_{2}$ on Project GAMETAG, Eos Trans. AGU, 59, 1081, 1978.

McElroy, M. B., S. C. Wofsy, and N. D. Sze, Photochemical sources for atmospheric $\mathrm{H}_{2} \mathrm{~S}$, Atmos. Environ., 14, 159-163, 1980.

Nguyen, B. C., Etude par les traceurs radioactifs des échanges entre les diverses zones de l'atmosphère au-dessus des continents et des océans, Ph.D. thesis, Fac. Sci. Paris, 1968.

Nguyen, B. C., and G. Lambert, Geographic variations of the largescale vertical exchange speed, J. Geophys. Res., 75, 2877-2884, 1970.

Nguyen, B. C., B. Bonsang, and G. Lambert, The atmospheric concentration of sulfur dioxide and sulfate aerosols over antarctic, subantarctic areas and ocean, Tellus, 26, 241-249, 1974a.

Nguyen, B. C., B. Bonsang, J. L. Pasquier, and G. Lambert, Composantes marines et africaines des aérosols de sulfates dans l'hémisphère sud, J. Rech. Atmos., 8, 831-844, $1974 b$.

Nguyen, B. C., B. Bonsang, G. Lambert, and J. L. Pasquier, Residence 
time of sulfur dioxide in the marine atmosphere, Pure Appl. Geophys., 123, 489-500, 1975.

Nguyen, B. C., A. Gaudry, B. Bonsang, and G. Lambert, Reevaluation of the role of dimethyl sulphide in the sulphur budget, Nature, 275, 637-639, 1978a.

Nguyen, B. C., and B. Bonsang, Transport of continental sulfur compounds over the Mediterranean, in Atmospheric Pollution 1978 edited by M. M. Benarie, pp. 201-205, Elsevier, New York, $1978 b$.

Ostlund, H. G., and J. Alexander, Oxidation rate of sulfide in seawater, A preliminary study, J. Geophys. Res., 68, 3995-3997, 1963.

Paugam, J. Y., B. C. Nguyen, B. Bonsang, and S. Fongang, Production de noyaux aitken et de composés soufrés dans l'air audessus d'une zone littorale, Chemosphere, 6, 333-339, 1977.

Prahm, L. P., U. Torp, and R. M. Stern, Deposition and transformation rates of sulphur oxides during atmospheric transport over the Atlantic, Tellus, 28, 355-372, 1976.

Rasmussen, R. A., Emission of biogenic hydrogen sulfide, Tellus, 26, 254-260, 1974.

Ravishankara, A. R., N. M. Kreutter, R. C. Shak, and P. H. Wine, Rate of reaction of $\mathrm{OH}$ with COS, Geophys. Res. Lett., 7, 861-864, 1980.

Robinson, E., and R. C. Robbins, Gaseous atmospheric pollutants from urban and natural sources, in Global Effects of Environmental Pollution, edited by S. F. Singer, D. Reidel, Hingham, Mass., 1970.

Rodhe, H., Measurements of sulfur in the free atmosphere over Sweden 1969-1970, J. Geophys. Res., 77, 4494-4499, 1972.

Rodhe, H., and I. Isaksen, Global distribution of sulfur compounds in the troposphere estimated in a height/latitude transport model, $J$. Geophys. Res., 85, 7401-7409, 1980.

Schiff, J. A., Sulfur, in Physiology and Biochemistry of Algae (Sulfur Halogens), edited by R. A. Lewin, pp. 239-246, Academic, New York, 1962.

Slagle, I. R., R. E. Graham, and D. Grutman, Direct identification of reactive routes and measurements of rate constants in the reactions of oxygen atoms with methanethiol, ethanethiol, and methylsulfide, Int. J. Chem. Kinet., 8, 451-458, 1976.
Slatt, B. J., D. R. Natush, J. M. Prospero, and D. L. Savoie, Hydrogen sulfide in the atmosphere of the Northern Equatorial Atlantic Ocean and its relation to the global sulfur cycle, Atmos. Environ., 12,981-991, 1978.

Smith, F. B., and G. H. Jeffrey, Airborne transport of sulfur dioxide from the UK, Atmos. Environ., 9, 643-659, 1975.

Stoiber, R. E., and A. Jepsen, Sulfur dioxide contribution to the atmosphere by volcanos, Science, 182, 577-578, 1973.

Sze, N. D., and M. K. W. Ko, Photochemistry of COS, $\mathrm{CS}_{2}$, $\mathrm{CH}_{3} \mathrm{SCH}_{3}$, and $\mathrm{H}_{2} \mathrm{~S}$ : Implications for the atmospheric sulfur cycle, Atmos. Environ., 14, 1223-1234, 1980.

Torres, A. L., P. J. Maroulis, A. B. Goldberg, and A. R. Bandy, Atmospheric carbonyl sulfide measurements on Project GAMETAG, J. Geophys. Res., 85, 7357-7360, 1980.

Varhelyi, G., On the vertical distribution of sulfur compounds in the lower troposphere, Tellus, 30, 542-545, 1978.

West, P. W., and G. C. Gaeke, Fixation of sulfur dioxide as disulfitomercurate (II) and subsequent colorimetric estimation, Anal. Chem., 28, 1816-1819, 1956.

Wine, P. H., W. L. Chameides, and A. R. Ravishankara, Potential role of $\mathrm{CS}_{2}$ photooxidation in tropospheric sulfur chemistry, Geophys. Res. Lett., 8, 543-546, 1981.

Woodwell, G. M., R. H. Whittacker, W. A. Reiners, G. E. Likens, C. C. Delwiche, and D. B. Botkin, The biota and the world carbon budget, Science, 199, 141-144, 1978.

B. Bonsang, A. Gaudry, and B. C. Nguyen, Centre des Faibles Radioactivités, Laboratoire Mixte CNRS-CEA, 91190 Gif-sur-Yvette, France.
(Received November 12, 1981; revised June 6, 1983; accepted June 21, 1983.) 